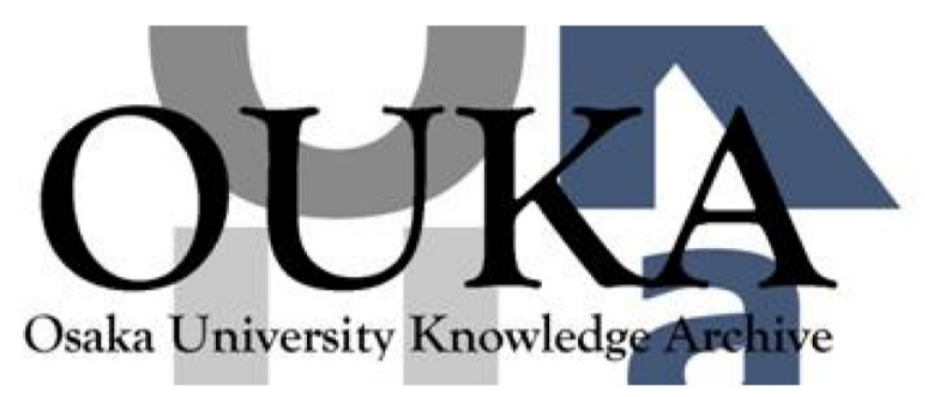

\begin{tabular}{|c|l|}
\hline Title & $\begin{array}{l}\text { Flow reversals in particle-dispersed natural } \\
\text { convection in a two-dimensional enclosed square } \\
\text { domain }\end{array}$ \\
\hline Author(s) & $\begin{array}{l}\text { Takeuchi, Shintaro; Miyamori, Yuri; Gu, } \\
\text { Jingchen et al. }\end{array}$ \\
\hline Citation & Physical Review Fluids. 4(8) p. 84304 \\
\hline Issue Date & $2019-08-21$ \\
\hline oaire:version & VoR \\
\hline URL & https://hdl.handle. net/11094/73149 \\
\hline rights & o2019 American Physical Society \\
\hline Note & \\
\hline
\end{tabular}

Osaka University Knowledge Archive : OUKA

https://ir. Library. osaka-u. ac. jp/

Osaka University 


\title{
Flow reversals in particle-dispersed natural convection in a two-dimensional enclosed square domain
}

\author{
Shintaro Takeuchi, Yuri Miyamori, Jingchen Gu, and Takeo Kajishima \\ Department of Mechanical Engineering, Osaka University, \\ 2-1 Yamada-oka, Suita-city, Osaka 565-0871, Japan
}

(Received 18 August 2018; published 21 August 2019)

\begin{abstract}
Flow reversals in natural convection of particle-dispersed two-phase flow in a twodimensional square box are studied by numerical simulation. The Rayleigh number based on the domain side length is set to $10^{4}$. The domain accommodates $11^{2}$ neutrally buoyant circular particles, and the thermal conductivity of the particle is set to $10^{2}$ times higher than the ambient fluid. The particle-dispersed flow driven by buoyancy develops into circulating flow, which transports particles into a diagonal pair of corner regions of the domain. The particles vertically aligned in the corner regions are a strong source of moment of buoyancy in the counterconvective direction, and flow reversals take place at the intervals of several hundred convective-time scales. The mechanism is different from that of the reversals or oscillation in single-phase or particle-dispersed natural convection reported in the literature. Thermal effect of the vertically aligned particles in a corner is modeled by nondimensionalized heat flux, and a cross-coupled sum of those of the four corners is found to be a precursor indicator of the reversal events. The investigation on the effects of three major parameters (Rayleigh number, conductivity ratio, and average interparticle spacing) suggests that the reversals occur in a small region in the parameter space.
\end{abstract}

DOI: 10.1103/PhysRevFluids.4.084304

\section{INTRODUCTION}

In a single phase buoyancy-driven flow between heated bottom and cooled top boundaries, known as a Rayleigh-Bénard (RB) cell, the balance between buoyancy and viscous forces breaks at a certain Rayleigh number and thermal convection sets in [1,2]. In a two-dimensional (2D) single-phase RB convection (Rayleigh number in the range between $10^{7}$ and $10^{8}$, and Prandtl number between 2.0 and 4.3), reversal of the main flow direction has been reported: the main vortices in the center region and corner regions exchange the energy and eventually the direction of natural convection is reversed [3-7]. In most reversal events, the reversal is consecutive and the period is regular, although it happens to accompany occasional cessation and "rogue" reversal [7]. Sugiyama et al. [4] and Chandra and Verma [6] mentioned the mechanism as momentum supply into the corner vortices by plumes detached from the horizontal boundary layers on the top and bottom walls, and Sugiyama et al. [4] summarized the reversal conditions in the parameter space. Podvin and Sergent [8] developed a low-dimensional model of reversals in a RB cell by a proper orthogonal decomposition (POD) analysis, and Castillo-Castellanos et al. [7] explained the reversals based on the energy balance. Several studies have suggested that the reversal dynamics is described by a probability resonance in a double-well system $[3,9,10]$, a symmetry recovering process of the forcing [11], and a generalized Lorentz attractor model $[12,13]$.

The present authors have reported similar consecutive reversals (or oscillation) in a 2D particledispersed RB cell at Rayleigh number $10^{4}$ [14]: a total of $N_{p}=14^{2}$ neutrally buoyant particles are initially arranged uniformly in a square domain (the solid volume fraction is $38 \%$ ), and numerical simulation predicted consecutive reversal modes of the solid-dispersed flow around the domain 
center when the thermal conductivity ratio of the solid to the fluid $\left(\lambda_{s} / \lambda_{f}\right)$ is much higher than unity. The reversal periods are well established with an amplitude of variation angle less than $2 \pi$, and therefore, the motions of the fluid and particles are rather recognized as "oscillation." During the oscillation, the particle arrangement retains the symmetry with respect to the domain center. Hereafter, this oscillation mode is referred to as the regular symmetric oscillation. The oscillation was explained by the restitution of the buoyancy force generated when the particles are slightly displaced from the initial neutral positions. A global conservation model of the angular momentum with infinitesimal displacement assumption showed that the oscillation period is scaled by onequarter power of $\lambda_{s} / \lambda_{f}[14]$ in a higher conductivity range $\left(10^{2} \leqslant \lambda_{s} / \lambda_{f} \leqslant 10^{3}\right)$. For triggering the regular symmetric oscillation mode, the difference in time scales for heat convection in the fluid phase and heat conduction in the solid phase (i.e., finite-size effect of the particle) was found to be essential [14]. This result suggests a limitation of interpreting the system as a homogeneous isotropic suspension (with a higher effective conductivity than that of the surrounding fluid) even in such a coherent system. Further, the local effect at the individual particle scale is even pronounced in a system of nonuniform particle distribution: the heat transfer is influenced by the particle size, local number density of particles, and so on [15], and the presence of particles also influences the effective viscosity [16].

Regular symmetric oscillation is still observed by decreasing $N_{p}$ to $13^{2}$ and $12^{2}$. By further decreasing it to $N_{p}=11^{2}$ (the solid volume fraction 23\%) while keeping the other parameters the same (including $\lambda_{s} / \lambda_{f}$ and the Rayleigh number), however, a different reversal pattern of a rather long and random period emerges. In the initial developing stage under this condition, a large-scale circulation mode evolves and the particle arrangement is no longer symmetric. However, the circulating flow reverses at nonregular intervals of several hundred convective-time units, which is much longer than the period of the regular symmetric oscillation. The extremely long period of time between the reversals (with variation angle more than $2 \pi$ ) and asymmetric (or uneven) particle arrangement at the instant of reversal suggest a different mechanism from the regular symmetric oscillation, and this is a reversal mode in a particle-dispersed RB convection.

In the present work, we study this nonregular reversal mode in a particle-dispersed natural thermal convection observed after the symmetry of particle arrangement breaks. Like the regular oscillation case, the time scale difference for heat transport through the fluid and solid phases is also essential in this case. This necessitates a treatment as an inhomogeneous suspension (at the individual particle scale) for interpreting the multiphase reversal events. We focus on the interplay between the moment of buoyancy and uneven distribution of corner particles, which occasionally causes reversal motions.

\section{GOVERNING EQUATIONS}

Throughout the present study, the following properties of the fluid and solid phases are assumed to be constant: density $\rho$, viscosity $\mu$, thermal conductivity $\lambda$, volumetric thermal expansion coefficient $\beta$, and specific heat $c$. The subscripts $f$ and $s$ indicate the fluid and solid phases, respectively.

The governing equations for the fluid are the continuity equation, Navier-Stokes equation with Boussinesq approximation, and the energy equation:

$$
\begin{gathered}
\nabla \cdot \boldsymbol{u}_{f}=0 \\
\frac{\partial \boldsymbol{u}_{f}}{\partial t}+\boldsymbol{u}_{f} \cdot \nabla \boldsymbol{u}_{f}=-\frac{\nabla p}{\rho_{f}}+\frac{\mu_{f}}{\rho_{f}} \nabla^{2} \boldsymbol{u}_{f}+\boldsymbol{g} \beta_{f}\left(T-T_{0}\right), \\
\frac{\partial T}{\partial t}+\boldsymbol{u}_{f} \cdot \nabla T=\frac{\lambda_{f}}{\rho_{f} c_{f}} \nabla^{2} T
\end{gathered}
$$

where $\boldsymbol{u}_{f}$ is the fluid velocity, $t$ is the time, $p$ is the static pressure, $\boldsymbol{g}$ is the gravitational acceleration, $T$ is the temperature, and $T_{0}$ is the reference temperature. 


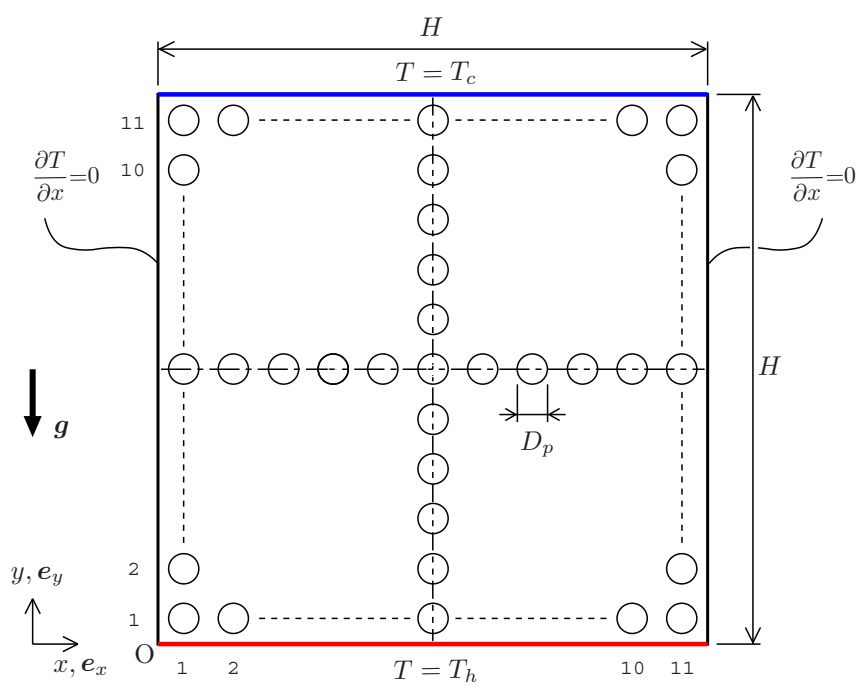

FIG. 1. Schematic of the arrangement of the particles in the square domain. The number of particles is $N_{p}=11^{2}$ unless specified otherwise (Sec. V).

Particles are monosized rigid circular objects. The particles are tracked individually in a Lagrangian way by solving the equations of translational and rotational motions:

$$
\begin{gathered}
m_{p} \frac{d \boldsymbol{u}_{t}}{d t}=\int_{A_{p}} \boldsymbol{\tau} \cdot \boldsymbol{n} d s+\boldsymbol{G}_{s}, \\
\boldsymbol{I}_{p} \cdot \frac{d \boldsymbol{\omega}}{d t}=\int_{A_{p}} \boldsymbol{r} \times(\boldsymbol{\tau} \cdot \boldsymbol{n}) d s+\boldsymbol{N}_{s},
\end{gathered}
$$

where $m_{p}$ is the mass of the particle, $\boldsymbol{u}_{t}$ is the translational velocity, $A_{p}$ is the particle surface, $\boldsymbol{\tau}$ is the fluid stress tensor, $\boldsymbol{n}$ is the outward unit normal vector at the particle surface, $\boldsymbol{G}_{s}$ is the external force, $\boldsymbol{I}_{p}$ is the inertia tensor of the particle, $\boldsymbol{\omega}$ is the angular velocity, $\boldsymbol{r}$ is the relative position vector from the gravity center of the particle, and $N_{s}$ is the moment of the external force. The energy conservation in the solid phase,

$$
\frac{\partial T}{\partial t}+\boldsymbol{u}_{s} \cdot \nabla T=\frac{\lambda_{s}}{\rho_{s} c_{s}} \nabla^{2} T
$$

is solved on the Eulerian frame together with Eq. (3), where $\boldsymbol{u}_{s}=\boldsymbol{u}_{t}+\boldsymbol{\omega} \times \boldsymbol{r}$.

\section{COMPUTATIONAL SETUP}

The computational domain is a square box of side length $H$ as shown in Fig. 1. The temperatures on the top and bottom walls are fixed to $T_{c}$ and $T_{h}$, respectively, and $T_{h}-T_{c}=\Delta T(>0)$ is kept constant. The lateral walls are thermally insulated. A total number of $N_{p}$ particles of diameter $D_{p}$ are initially arranged uniformly in both $x$ and $y$ directions.

Hereafter, the variables are nondimensionalized by the reference length $H$, the characteristic temperature difference $\Delta T$, the reference velocity $U=\sqrt{g \beta_{f} \Delta T H}$, and the reference pressure $\rho_{f} U^{2}$. The Rayleigh number and Prandtl number are as follows:

$$
\mathrm{Ra}=\frac{g \beta_{f} \Delta T H^{3}}{\nu_{f} \kappa_{f}}, \quad \operatorname{Pr}=\frac{\nu_{f}}{\kappa_{f}},
$$

where $g=|\boldsymbol{g}|, \nu_{f}=\mu_{f} / \rho_{f}$ and $\kappa_{f}=\lambda_{f} /\left(\rho_{f} c_{f}\right)$. 
TABLE I. Nondimensional numbers and computational parameters for Sec. IV.

\begin{tabular}{lcc}
\hline \hline Rayleigh number & $\mathrm{Ra}$ & $10^{4}$ \\
Prandtl number & $\mathrm{Pr}$ & 1 \\
Reference temperature & $T_{0} / \Delta T$ & $1 / 2$ \\
Density ratio & $\rho_{s} / \rho_{f}$ & 1 \\
Volumetric thermal expansion ratio & $\beta_{s} / \beta_{f}$ & 1 \\
Specific heat ratio & $c_{s} / c_{f}$ & 1 \\
Thermal conductivity ratio & $\lambda_{s} / \lambda_{f}$ & $10^{2}$ \\
Number of particles & $N_{p}$ & $11^{2}$ \\
Number of grid points & $N_{x} \times N_{y}$ & $200 \times 200$ \\
Time increment & $\Delta t U / H$ & $1.0 \times 10^{-4}$ \\
Diameter of particles & $D_{p} / H$ & 0.05 \\
\hline \hline
\end{tabular}

The governing equations for the fluid phase are solved on a fixed Cartesian coordinate system. The second-order Adams-Bashforth and Crank-Nicolson methods are employed for the convective and viscous terms, and a fractional step method is used for coupling the incompressible velocity and pressure fields. The fluid-solid interaction is solved by an immersed solid method [17] with a two-stage coupling scheme [18]. The continuity of the heat flux at the fluid-particle interface is guaranteed by the interfacial heat flux model [14].

For tracking the particles, a discrete element method with a soft-sphere collision model [19] is employed.

\section{RESULTS AND DISCUSSION}

After explaining the typical motions of the fluid and particles in particle-dispersed RB convection, we introduce some characteristic quantities for the circulating and reversal modes. The values employed for the nondimensional numbers and computational parameters for this section are summarized in Table I.

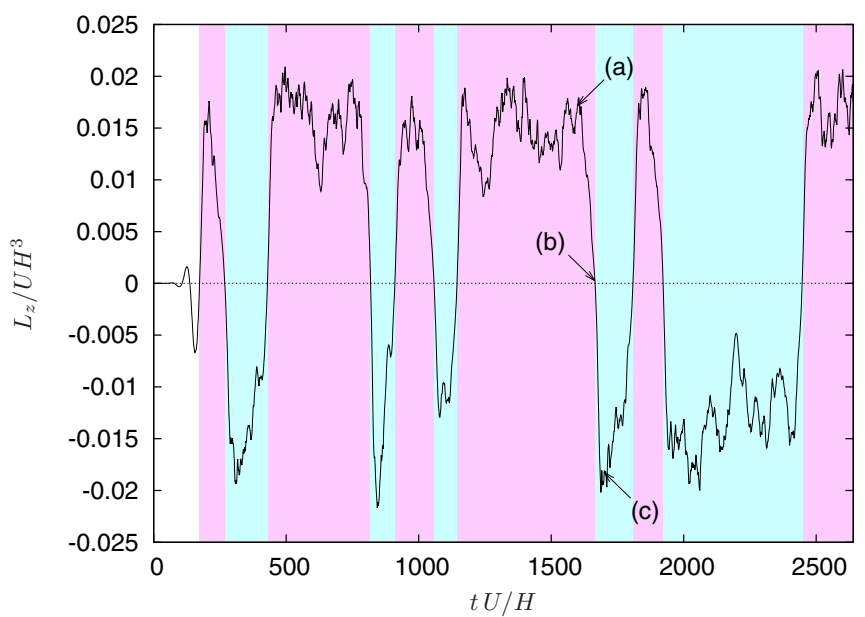

FIG. 2. Time history of the angular momentum around the domain center, $L_{z}$. The background colors represent clockwise (cyan) and counterclockwise (magenta) rotations. Typical three instants (a) before (b) at and (c) after a reversal are indicated in the figure. 


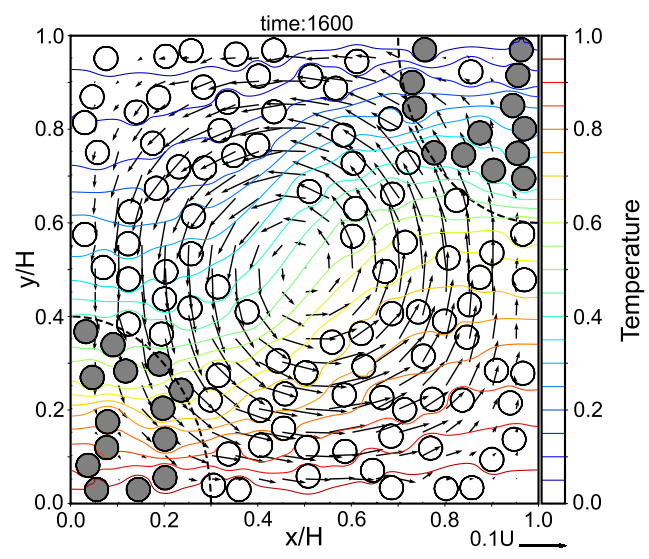

(a) $t U / H=1600$

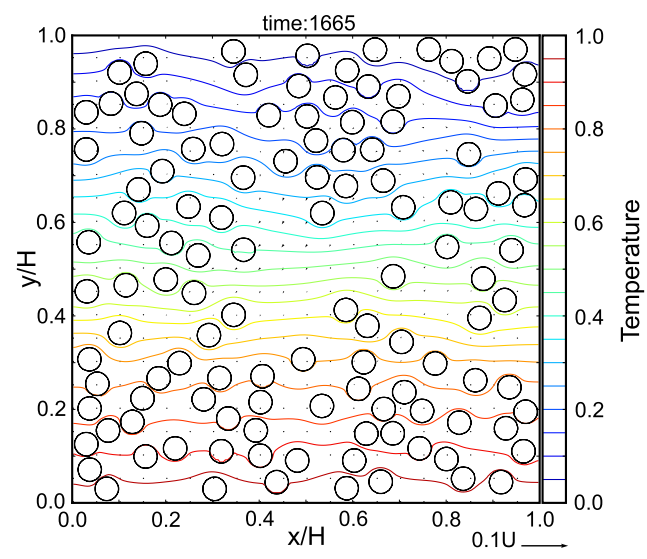

(b) $t U / H=1665$

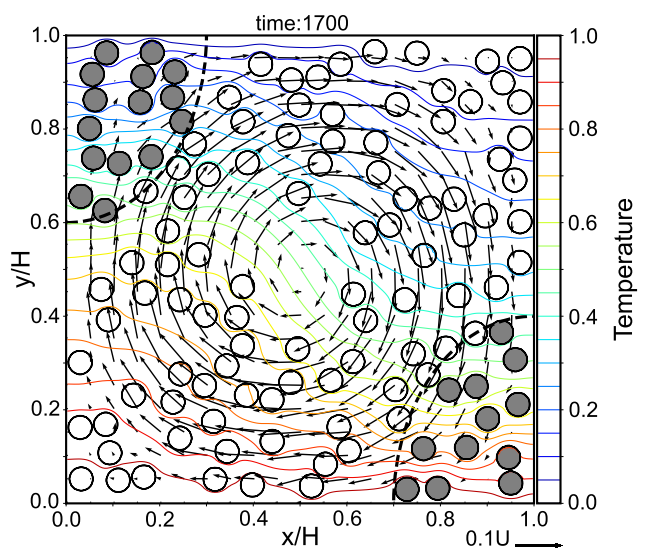

(c) $t U / H=1700$

FIG. 3. Velocity and temperature fields overlapped with the particle arrangements at three different times (a) before reversal, (b) of reversal, and (c) after reversal, which correspond to the instants indicated in Fig. 2. The particles aligned in the counterconvective corners are highlighted (see Sec. IV C). The velocity vectors are plotted at every 20 grid point in both $x$ and $y$ directions.

\section{A. Time history of the angular momentum}

To characterize the circulating flow around the domain center, the angular momentum of the system is introduced:

$$
L_{z}(t)=\int\left[-\left(y-\frac{H}{2}\right) u_{x}+\left(x-\frac{H}{2}\right) u_{y}\right] d x d y
$$

where $u_{x}$ and $u_{y}$ are, respectively, the $x$ and $y$ components of the volume-averaged velocity field, $\alpha \boldsymbol{u}_{s}+(1-\alpha) \boldsymbol{u}_{f}$, where $\alpha$ is the local solid volume fraction. Figure 2 shows the time history of $L_{z}$. A positive value indicates that the convection is in the counterclockwise direction, and a clockwise convection takes a negative value. When $L_{z}$ is 0 , the system takes either a stationary state or a multiple-vortex state making up the zero angular momentum in total. The background colors in the figure, cyan and magenta, correspond to the clockwise and counterclockwise convection, respectively. The figure shows that the convection direction is reversed repeatedly. However, no clear periodicity can be detected. 


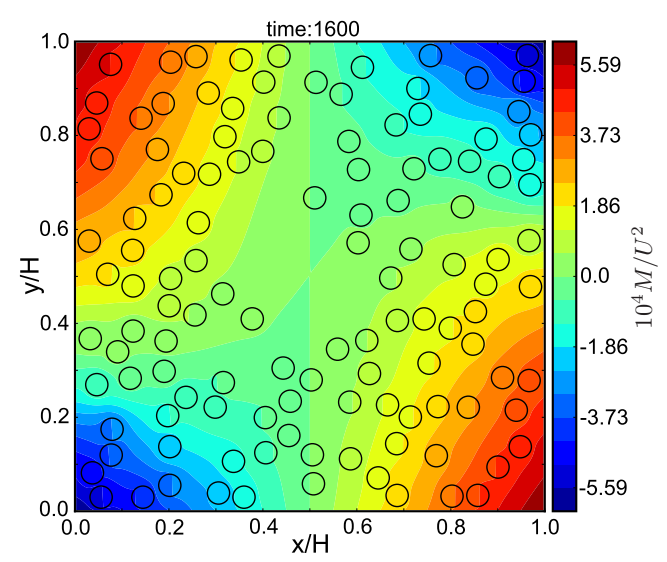

(a) $t U / H=1600$

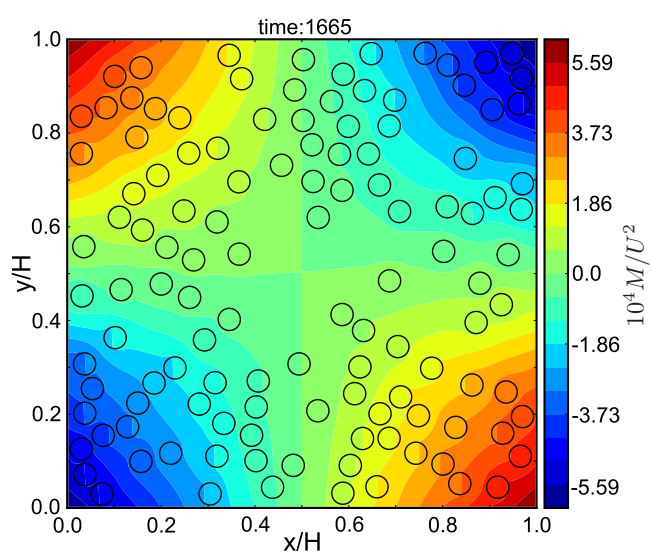

(b) $t U / H=1665$

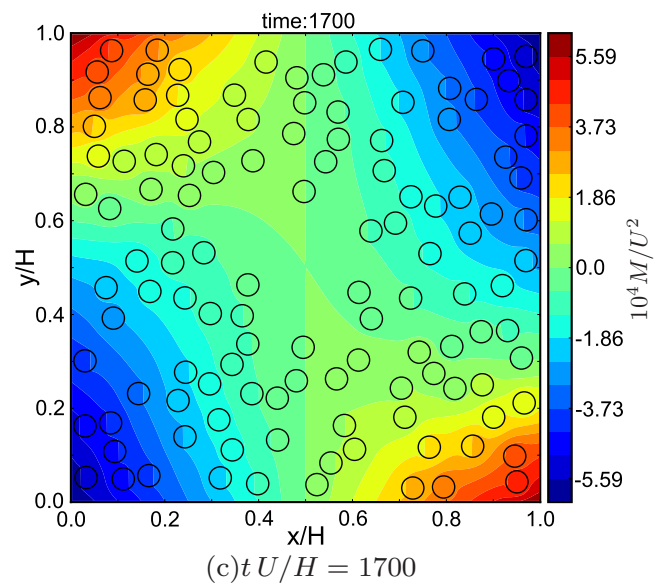

FIG. 4. Typical distributions of moment of buoyancy, $M(x) / U^{2}$, at three different instants (a)-(c) indicated in Fig. 2.

The points (a), (b), and (c) indicated in Fig. 2 are the representative instants around a reversal event; at (a) and (c), counterclockwise and clockwise convections are predominant, respectively, and (b) shows the instant of reversal between (a) and (c). Figure 3 shows the snapshots of the velocity and temperature fields at those instants together with the particle outlines. The velocity vectors and temperature are represented by arrows and contours, respectively. In either convection state, a single vortex exists around the domain center, while, at the instant of the reversal, both the fluid and particles are stationary and temperature contours are almost horizontal. We confirmed in our preliminary study that the other instants of the reversal show the similar velocity and temperature fields.

Considering that the reversals in a single phase $2 \mathrm{D} \mathrm{RB}$ convection $\left(\mathrm{Ra}>10^{6}\right)$ is due to the development of multiple vortices in the center and corner regions [4,5,7], the above results clearly show that the reversals of the solid-dispersed RB convection are caused by a different mechanism from those of the single phase RB convection in terms of the flow structure. On the other hand, the mingled particle patterns in Fig. 3 also suggest a different mechanism from the regular symmetric oscillation. Recalling that the regular oscillation periods have ranged between $30 H / U$ and $40 H / U$ [14], the extremely long periods in Fig. 2 also suggest that the infinitesimal displacement model for the regular symmetric oscillation does not apply. 


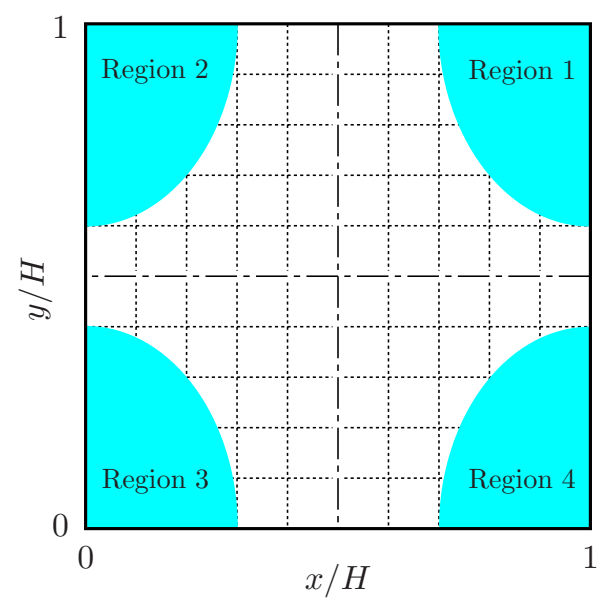

FIG. 5. Corner region is defined as elliptic region of minor and major axes of $0.3 H$ and $0.4 H$ in the $x$ and $y$ directions, respectively. Labelling of the corner regions is the same as the quadrants.

\section{B. Moment of buoyancy (MoB) in corner regions}

We focus on distribution of moment of buoyancy $(\mathrm{MoB})$. Due to the Boussinesq approximation, the MoB is defined as

$$
M(\boldsymbol{x})=g \beta\left(T(\boldsymbol{x})-T_{0}\right)\left(x-\frac{H}{2}\right) .
$$

Figure 4 shows the distribution of the MoB at $t U / H=$ (a) 1600 , (b) 1665, and (c) 1700 indicated in Fig. 2. When the counterclockwise convection is predominant [Fig. 4(a)], a strong MoB extends vertically in the top-left and bottom-right corners. In the following, the quarter-elliptic region of the major and minor axes $0.4 H$ and $0.3 H$, respectively, in each corner is focused. By following the labeling rule of the four corner regions in Fig. 5 (i.e., corners 1-4, the same labeling rule as the quadrants), the MoB in corner 2 (top left) and corner 4 (bottom right) [hereafter, the $(2,4)$ corners] drives the counterclockwise convection [Fig. 4(a)], and the clockwise convection is driven by the MoB in the $(1,3)$ corners (i.e., top right and bottom left) as Fig. 4(c) shows. On the other hand, at the instant of the reversal [Fig. 4(b)], the MoBs in the four corners exhibit about equal strengths, suggesting that the stationary state at the reversal instant [Fig. 3(b)] results from the balance of the MoBs in the $(2,4)$ and $(1,3)$ corners.

By evaluating the following absolute rate of $\mathrm{MoB}$ in the corner regions:

$$
M_{r}=\frac{\sum_{\ell=1}^{4} \int_{\text {Corner } \ell}\left|g \beta_{f}\left(T-T_{0}\right)(x-H / 2)\right| d S}{\int_{\text {Entire region }}\left|g \beta_{f}\left(T-T_{0}\right)(x-H / 2)\right| d S},
$$

the corner regions generate approximately $70 \%$ (in average) of the total absolute MoB over the domain, as shown in Fig. 6, except immediately after the initial stage of the simulation.

The above result suggests that the temporal variation of the MoB in the corner regions plays a key role to determine the direction of convection. Here, we define the total amount of the MoB in the corner regions (hereafter, the corner $\mathrm{MoB}$ ) as

$$
M_{\mathrm{cnr}}=\sum_{\ell=1}^{4} \int_{\text {Corner } \ell} g \beta_{f}\left(T-T_{0}\right)\left(x-\frac{H}{2}\right) d x d y \quad(\ell=1, \ldots, 4) .
$$

Figure 7 shows the time history of the corner MoB together with that of the angular momentum. Both $M_{\mathrm{cnr}}$ and $L_{z}$ are normalized by the respective maximum absolute values in the entire time range. The background color strips are the same as those in Fig. 2. Although the correlation is 


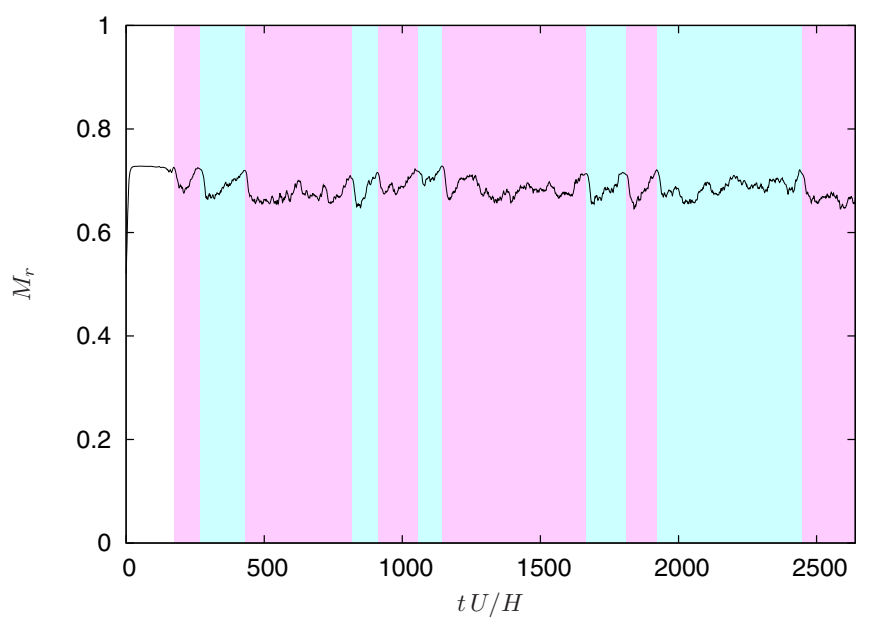

FIG. 6. The absolute sum of the moments of buoyancy in the four corners normalized by the absolute value of the moment of buoyancy in the entire domain. See Eq. (10). The colored background is the same as Fig. 2.

strong, careful observation infers that the switch of the convection direction is triggered when the sign of the corner MoB is reverted.

In the following, we show that this deviation of the corner MoB is induced by the conductive particles heated or cooled near the bottom or top wall, and the effect of the motion of the individual particles on the deviation of the corner MoB is investigated.

\section{Vertical alignment of particles}

As the incoming or outgoing particles to or from the corner regions would influence the heat transfer, time evolution of the number of corner particles is investigated. Figure 8 shows the time histories of the number of particles in the $(2,4)$ and $(1,3)$ corners. The raw data in Fig. 8(a) exhibit a slightly too noisy trend. Therefore, the following moving average is applied to the time history of

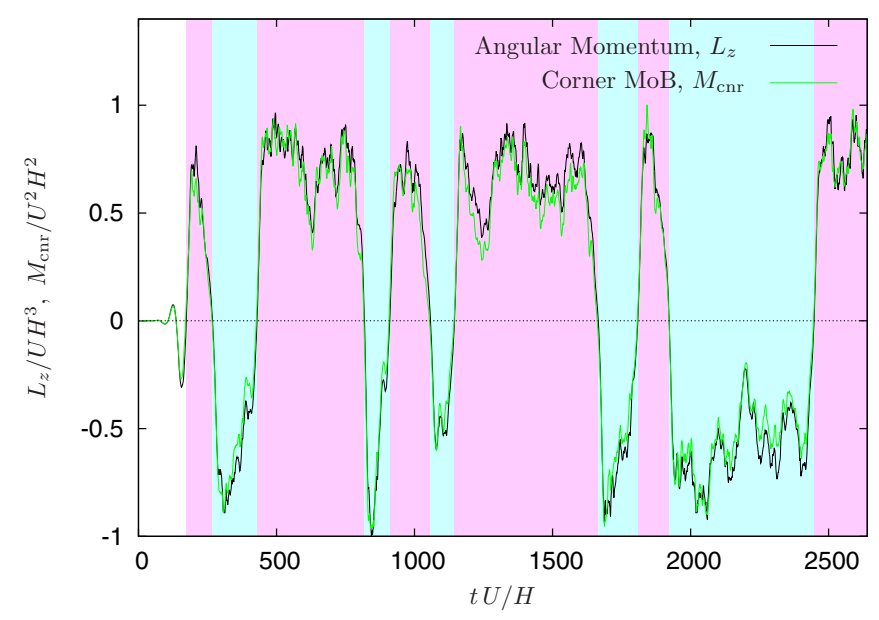

FIG. 7. Time histories of the corner moments of buoyancy $\left(M_{\mathrm{cnr}}\right)$ and the angular momentum $\left(L_{z}\right)$ around the domain center. Both are normalized by the respective absolute maximum values in the entire time range. The colored background is the same as Fig. 2. 


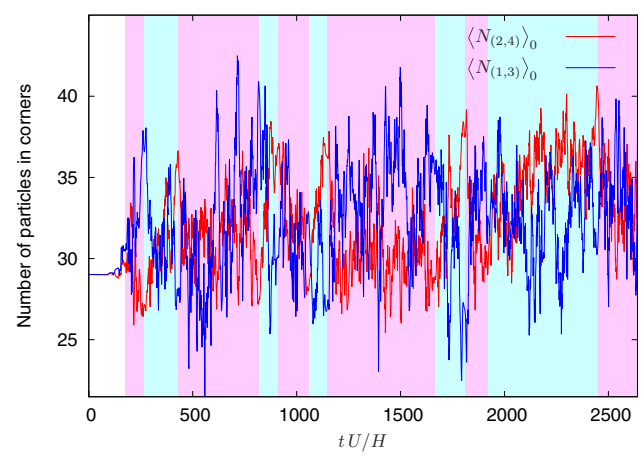

(a)

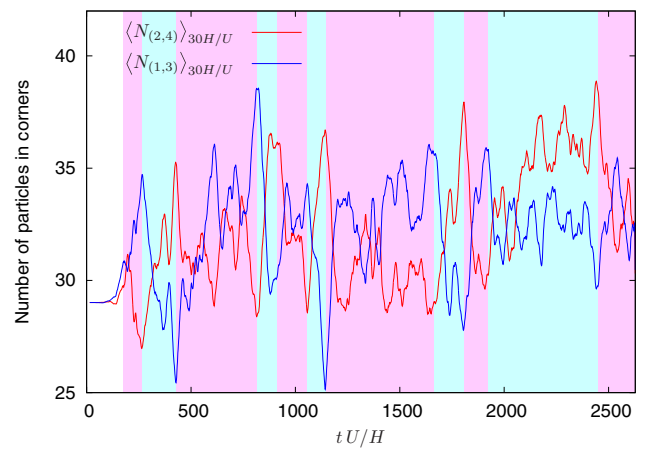

(c)

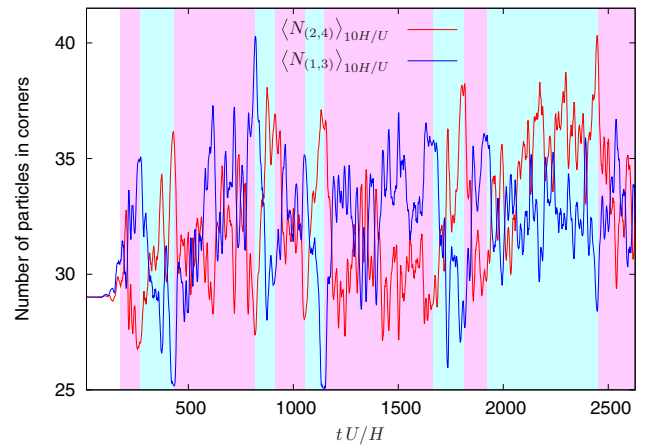

(b)

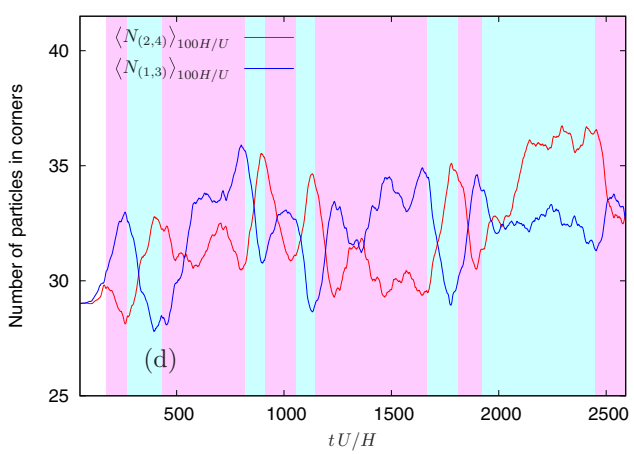

(d)

FIG. 8. Time history of the number of particles in corner regions. (a) Raw data, and the moving-averaged data with $\tau U / H=$ (b) 10 , (c) 30 , and (d) 100 .

the number of particles:

$$
\left\langle N_{(\ell, \ell+2)}\right\rangle_{\tau}(t)=\frac{1}{\tau} \int_{t-\tau / 2}^{t+\tau / 2} N_{(\ell, \ell+2)}(s) d s,
$$

where $N_{(\ell, \ell+2)}(\ell=1,2)$ is the number of particles in the $(\ell, \ell+2)$ corners. Figures $8(\mathrm{~b})-8(\mathrm{~d})$ are the filtered data with $\tau U / H=10,30$, and 100 . The case with $\tau U / H=30$ sufficiently resolves the increase and decrease trends in the respective pair of corners, while $\tau U / H=100$ oversmooths the profile particularly in the range between $t U / H=1800$ and 2400, which will be mentioned later. The graph shows that, in most cases, the numbers of particles in the two corner pairs deviate in the opposite phase, and the number of particles increases (on average) in the $(2,4)$ pair of corners in the clockwise state [e.g., Fig. 3(c)], while more particles are transported and accumulated in the $(1,3)$ pair of corners in the counterclockwise state [e.g., Fig. 3(a)]. Hereafter, those paired corners with inclined deposition trend of particles by the corresponding convections are referred to as counterconvective corners, and the complementary corner pairs are termed as driving corners. The driving corner means that the particles in the $(1,3)$ and $(2,4)$ corners work to accelerate the counterclockwise and clockwise convections, respectively.

The distributions of $M(x)$ in Figs. 4(a) and 4(c) suggest that the MoB in the respective driving corners is predominant. However, as the particles are transported to the counterconvective corners, it is expected that the apparent thermal conductivity in the corners tends to be high, eventually resulting in an overwhelming contribution to the counteracting MoB against the convection. This 
trend is even pronounced when the particles are aligned vertically so as to establish chained structure (viz., the interparticle conduction) toward the region away from the hot or cold wall [15].

In order to quantify the effect of the corner particles aligned in the vertical direction, the following cross-coupled weighted vertical alignment (WVA) index $\Xi$ is proposed:

$$
\Xi(t)=\left(\Xi_{2}+\Xi_{4}\right)-\left(\Xi_{1}+\Xi_{3}\right)
$$

where $\Xi_{\ell}(\ell=1,2,3,4)$ is the regional WVA index of the particles in corner $\ell$ defined as

$$
\Xi_{\ell}(t)=\sum_{\text {Eq. (15) }} w_{i, j}\left|\frac{\boldsymbol{r}_{i, j} \cdot \boldsymbol{e}_{y}}{D_{p}}\right| \quad(\ell=1, \ldots, 4),
$$

which represents the normalized heat flux based on the apparent thermal conductivity (due to the presence of the particles) in the region $\ell$, as detailed in the Appendix. Here, $\boldsymbol{e}_{y}$ is the unit vector in the vertically upward direction $(y)$. The relative vector between the particle centers $\boldsymbol{r}_{i, j}$ is defined as, $\boldsymbol{r}_{i, j}=\boldsymbol{x}_{j}^{p}-\boldsymbol{x}_{i}^{p}$, where $\boldsymbol{x}_{i}^{p}$ and $\boldsymbol{x}_{j}^{p}$ are the position vectors of the centers of the particles $i$ and $j$, respectively. The weight $w_{i, j}$ is given by either Eq. (A2) or Eq. (A6) depending on whether the conduction is dominant (i.e., Cond-Only) or both conduction and convection are influential (i.e., Cond + Conv). The summation is taken under the following condition:

$$
\left|\boldsymbol{r}_{i, j}\right|<2 D_{p} \text { and } \boldsymbol{r}_{i, j} \cdot \boldsymbol{e}_{y}>0 \text { in region } \ell .
$$

Note that $\Xi_{\ell}$ is always positive. A positive $\Xi_{\ell}$ in the top-left or top-right corner means that the heat is transferred in the vertically upward direction to the cold wall, and, as a result, the buoyancy works in the vertically downward direction. Therefore, the sum of $\Xi_{\ell}$ in the counterconvective corners models a counteracting MoB against the convection. The cross-coupled sum of $\Xi_{\ell}(\ell=$ $1, \ldots, 4)$, Eq. (13), accounts for the heat-transferring tendency around the domain center, which is one of the measures of the reversal potentiality. On the other hand, the temperature gradient in the horizontal direction is insensitive as observed in Fig. 3(b), and therefore, the horizontal alignment of particles has less influential effect for generating buoyancy and, in turn, the MoB in each corner region. Therefore, a positive $\Xi$ value represents that the particles in the $(2,4)$ corners contributes to the counterclockwise circulation $\left(\Xi_{2}+\Xi_{4}>\Xi_{1}+\Xi_{3}\right)$, whereas a negative value suggests that a larger number of particles are lined up in the vertical direction in the $(1,3)$ corners generating a predominant MoB in the clockwise direction.

In the following, the particle arrangement and reversal of convection are studied based on the WVA index. As observed in Fig. 8, the effect of incoming or outgoing particles appears as noisy fluctuation, and the same trend is observed in the time evolution of $\Xi(t)$. Therefore, the following moving average is applied:

$$
\langle\Xi\rangle_{\tau}(t)=\frac{1}{\tau} \int_{t-\tau / 2}^{t+\tau / 2} \Xi(s) d s .
$$

From our preliminary study, the case of $\tau U / H=30$ provides sufficiently good resolution to discuss the trend of the reversals for both WVA indices [with either Eq. (A2) or Eq. (A6)], and thereafter, the WVA index is shown with the above smoothing parameter. The sensitivity of $\tau$ on the resolution of the WVA index is addressed later.

The WVA indices are plotted in Fig. 9 together with the corner MoB, $M_{\mathrm{cnr}}$. Right before the direction of the convection switches, the absolute value of the WVA indices (both Cond-Only and Cond+Conv) take the prominent peaks (i.e., a local maximum or minimum), suggesting that the particles drift into the counterconvective corners and align in the vertical direction to generate a sufficient counteracting MoB against convection. Figure 10 compares the particle arrangements and the corresponding $M(\boldsymbol{x})$ at around the consecutive two reversal instants, where the particles in the counterconvective corners are highlighted by the following rule: when the centers of two particles (particle IDs $i$ and $j$ ) are in a corner region and the center-to-center distance satisfies $\left|\boldsymbol{r}_{i, j}\right|<2 D_{p}$, then both particles are highlighted. As shown in Figs. 10(a) and 10(b) for the reversal instant of $t U / H=815$, the convection is weak and the moment of buoyancy evenly distributes in 


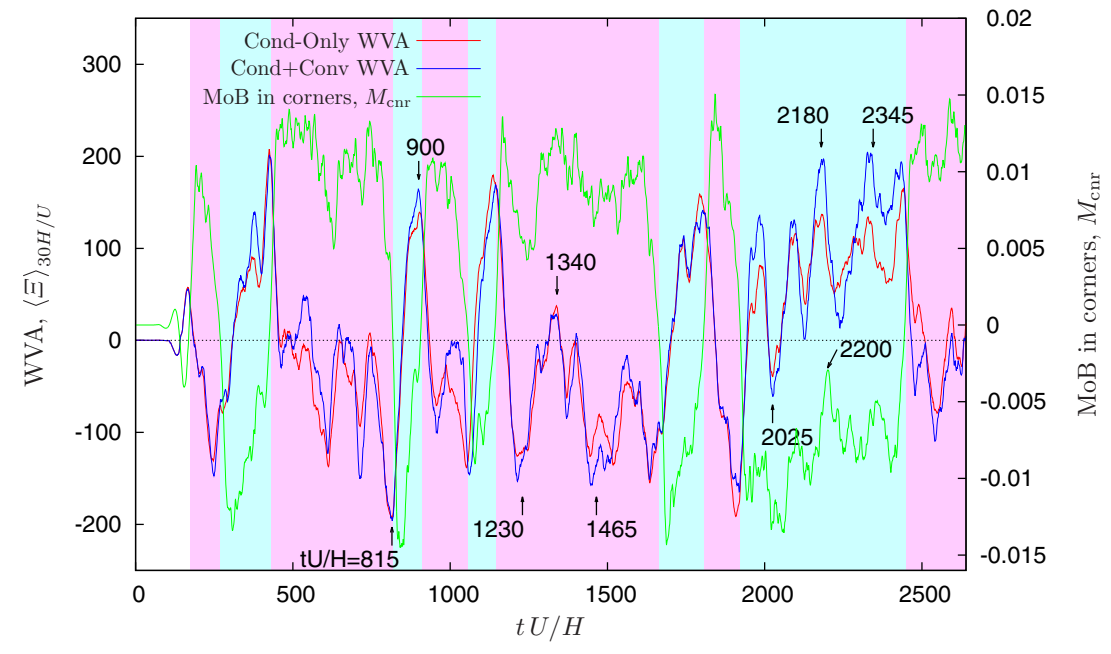

FIG. 9. Comparison of the WVA index (conduction only and conduction+convection with $\tau=30 \mathrm{H} / U$ ) with the corner moment of buoyancy, $M_{\mathrm{cnr}}$. In the figure, Cond-Only WVA denotes Eqs. (14) and (A2), and Cond+Conv WVAs refers to Eqs. (14) and (A6). Some characteristic events are labeled with time.

the four corners. Then, followed by the reversal into the clockwise direction, some particles are transported into the counterconvective corners, and just before the next reversal at $t U / H=900$ [Figs. 10(c) and 10(d)], a sufficient number of particles in the counterconvective corners generate a strong counteracting MoB (see Fig. 9). For the other reversal instants, the similar patterns of the flow, particle, and MoB are observed.

From the above result, the time of the prominent peak of the WVA advances to the sign switch of $L_{z}$ or $M_{\mathrm{cnr}}$. Here, the effective time advancement of the WVA to the reversal is investigated. Figure 11 plots the time histories of the WVA index on the shifted-time axis $t_{\text {shf }}$, together with $L_{z}$ and $M_{\mathrm{cnr}}$ which are identical to those in Fig. 7. The WVA index $\langle\Xi\rangle_{\tau}(\tau U / H=30)$ is time shifted around the instant of reversal $\left(t_{\mathrm{shf}}=0\right.$ when $\left.L_{z}=0\right)$, and each $\langle\Xi\rangle_{\tau}$ profile is normalized by its local maximum within the shifted time range $\left(-50 \leqslant t_{\mathrm{shf}} U / H \leqslant 50\right)$. Note that all the $\langle\Xi\rangle_{\tau}, L_{z}$, and $M_{\mathrm{cnr}}$ are displayed as the reversal from the clockwise to the counterclockwise directions; for the opposite reversal cases, the variables are multiplied by a negative sign. Over the ten reversal events in the entire time range studied, the average occurrence time of the maximum WVA index is found to be $\overline{\left.t_{\mathrm{shf}}\right|_{\max \langle\Xi\rangle_{30 H / U}}} U / H=-9.56$, and the WVA peaks are always in advance of the reversal instant. Our preliminary study shows that this result is insensitive to the selection of $\tau$ in the range of $10 \leqslant$ $\tau U / H \leqslant 50$ as $\overline{\left.t_{\mathrm{shf}}\right|_{\max \langle\Xi\rangle_{\tau}}} U / H=-9.57,-9.96,-15.60$, and -22.77 for $\tau U / H=10,50,70$, and 100, respectively. Therefore, the WVA of particles is a precursor index of a reversal event.

Particles are occasionally accumulated in driving corners. At $t U / H=1340$ when $\Xi$ takes the same sign as $M_{\mathrm{cnr}}$ (see Fig. 9), the particles are transported in the driving corners (as well as the counterconvective corners), as shown in Fig. 12. This formation of corner particles works to strengthen the magnitude of $M_{\mathrm{cnr}}$. The same strengthening formation is observed at around $t U / H=$ 2025. More generally, this reinforced-driving configuration is typically observed immediately after a reversed flow sets in. However, the particle configurations at $t U / H=1340$ and 2025 are not relevant to the preceding reversal, but those are realized by coincidence.

Throughout the studied time range of Fig. 9, the difference between Eqs. (A2) and (A6) is small in most occasions. This suggests that the effect of the convection on the WVA is relatively insignificant. However, there are some exceptions in certain time ranges: particularly in $1800 \leqslant t U / H \leqslant 2400$, for example, the flow direction does not switch even under prominent WVA values of the opposite sign to $M_{\mathrm{cnr}}$. In the next subsection, the effect of the convection is focused for these nonreversal events. 


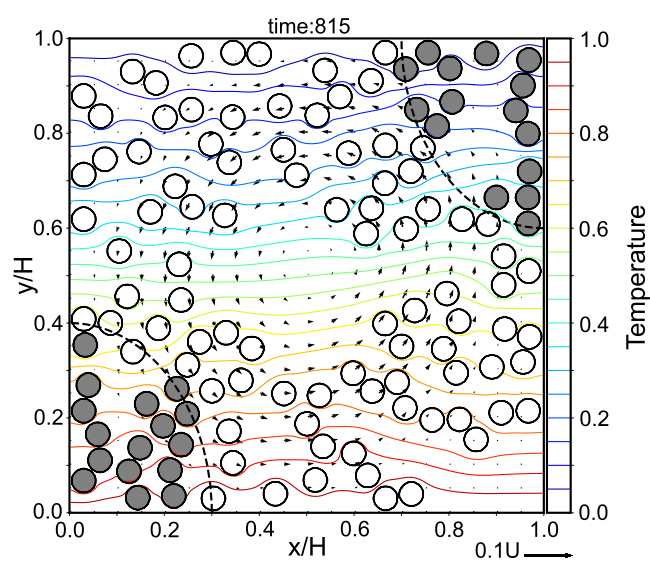

(a)

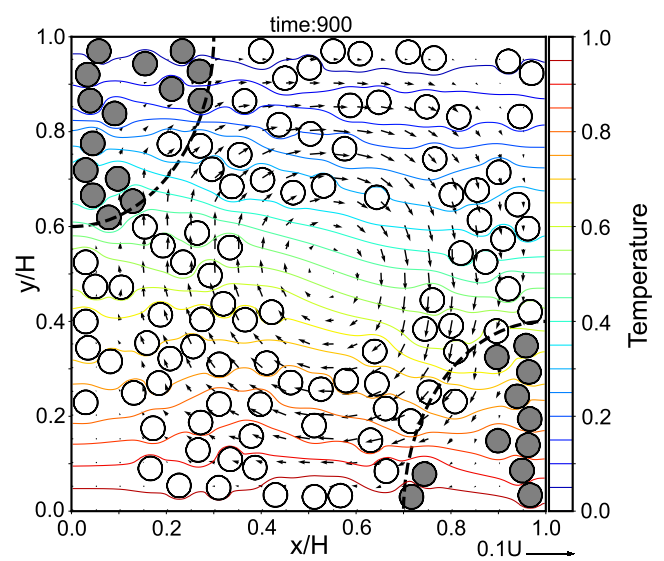

(c)

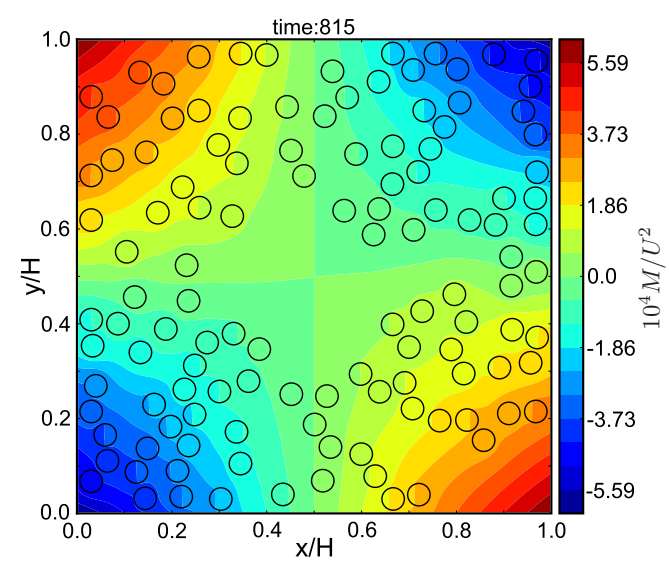

(b)

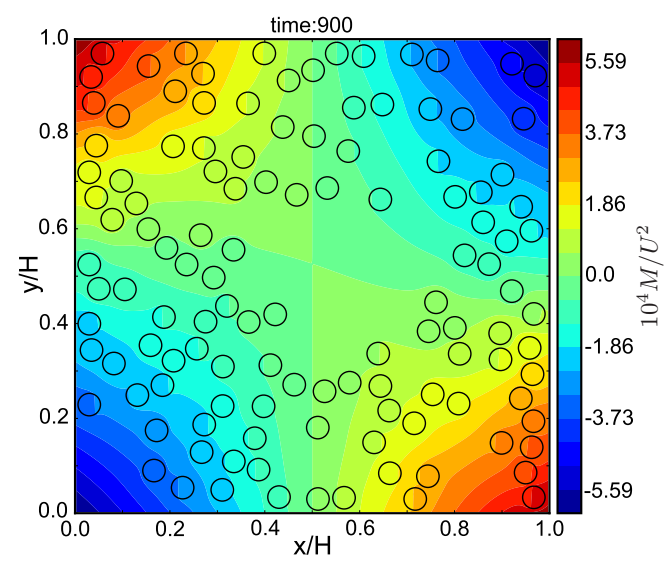

(d)

FIG. 10. Instantaneous flow fields of the temperature and velocity fields and moment of buoyancy at $t U / H=815$ and 900. The highlighted particles are selected as follows: when the centers of two particles (particle IDs $i$ and $j$ ) are in a corner region and the center-to-center distance satisfies $\left|\boldsymbol{r}_{i, j}\right|<2 D_{p}$, then both particles are highlighted.

\section{Nonreversal configurations associated with local maxima of WVA}

Vertical alignment of particles in counterconvective corners does not always induce flow reversal. For example, at $t U / H=1230,1465,2180$, and 2345 (and some other times) in Fig. 9, the WVA indices exhibit significant peaks, although the corresponding counteracting MoBs are not strong enough to complete the reversal.

Figure 13 shows the instantaneous contours of the velocity magnitude overlapped with the velocity vectors and the particle outlines at the above four times. Only the particles in the region outside the lowest contour of the velocity magnitude are highlighted in the figure, as those particles generate high-WVA index in the counterconvective corners. For the respective times, the numbers of particles in the counterconvective corners are not even: more particles assemble in one of the counterconvective corners than the other and the driving corners. Figure 14 shows the time evolutions of the regional WVA indices $\Xi_{\ell}(\ell=1,2,3,4)$ corresponding to Fig. 13, suggesting that the following regional WVA indices have significant values: $\Xi_{1}$ at $t U / H=1230, \Xi_{1}$ at $t U / H=1465, \Xi_{2}$ at $t U / H=2180$, and $\Xi_{2}$ at $t U / H=2345$. Despite the presence of the particles in the counterconvective corners, the circulating flow is not weak, 


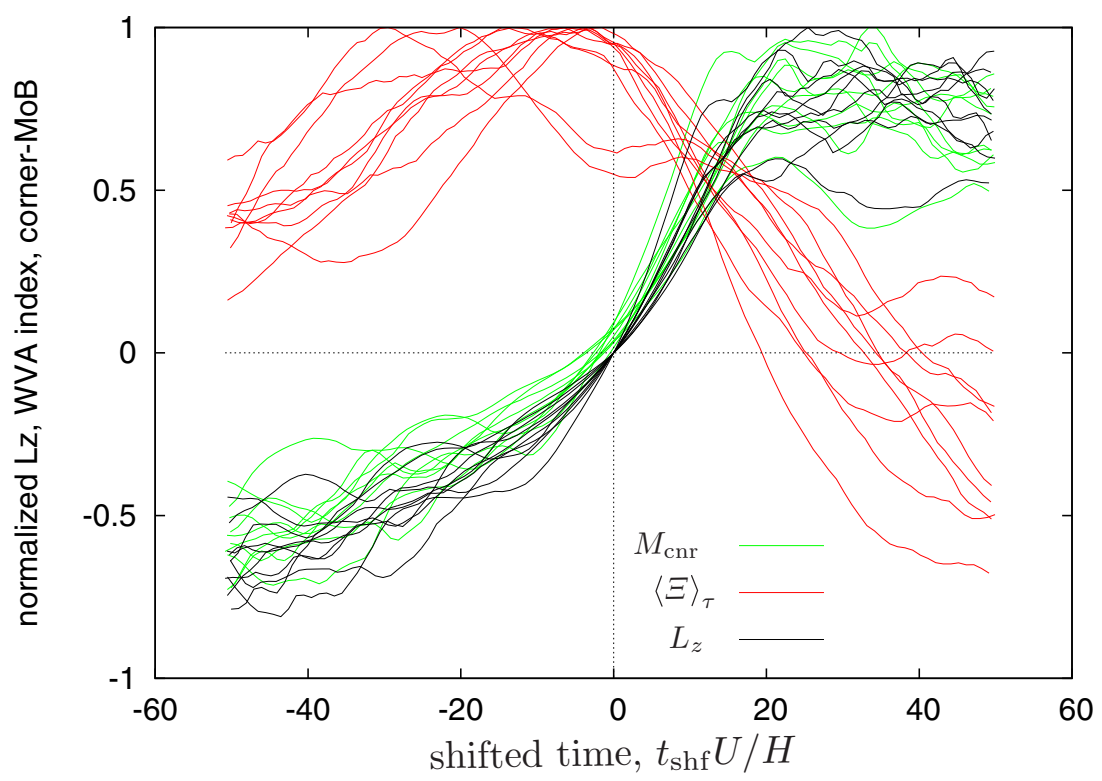

FIG. 11. The time histories of the Cond-Only WVA index time-shifted around the instants of the reversals, $t_{\text {shf }}=0$. The WVA index is normalized by its local maximum within the shifted time range $\left(-50 \leqslant t_{\text {shf }} U / H \leqslant\right.$ 50). Also plotted are the normalized $L_{z}$ and $M_{\mathrm{cnr}}$ (identical to those in Fig. 7), and all the plotted variables are displayed as the reversals from clockwise to counterclockwise directions by multiplying a negative sign for the cases of reversal from the counterclockwise to the clockwise directions.

but slightly shifted off center of the domain as if it avoids the biased counterconvective corner accommodating more particles. For regular reversal instants, on the other hand, the vortex center is located approximately at the center of the domain, as typically observed in Figs. 3 and 12(a).

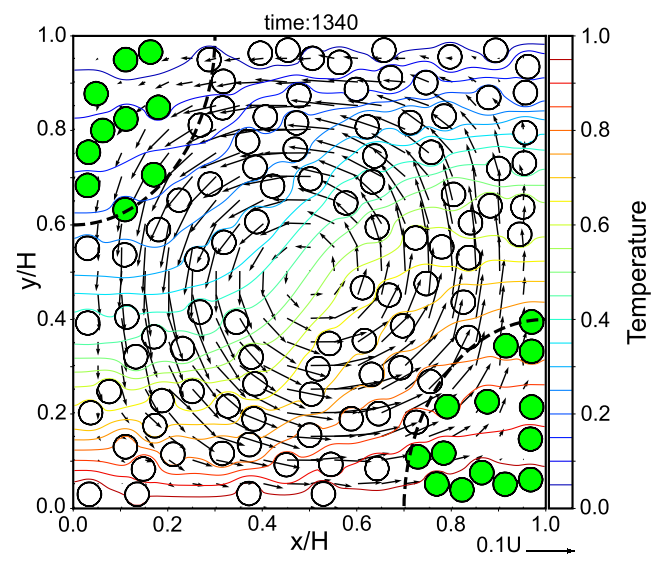

(a)

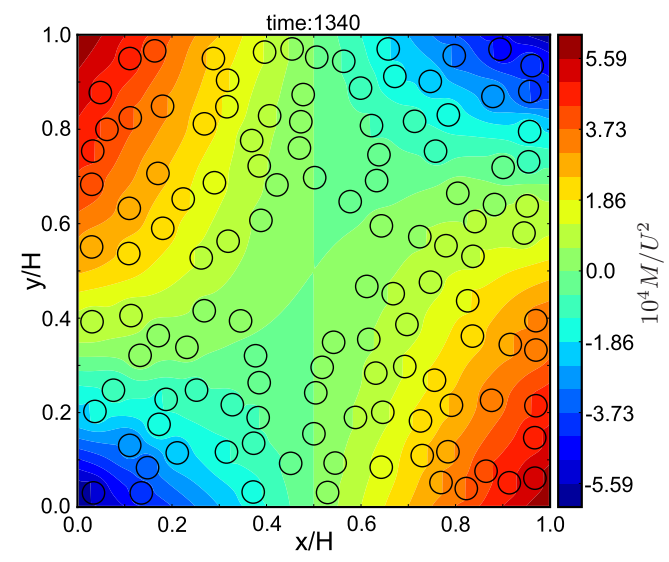

(b)

FIG. 12. Instantaneous flow field at $t U / H=1340$, (a) the temperature and velocity fields, (b) moment of buoyancy. The particles in the $(1,3)$ corners weaken the counterclockwise convection, while the particles in the $(2,4)$ corners accelerate the convection. The selection of the colored particles follows the same criterion as Fig. 10. 


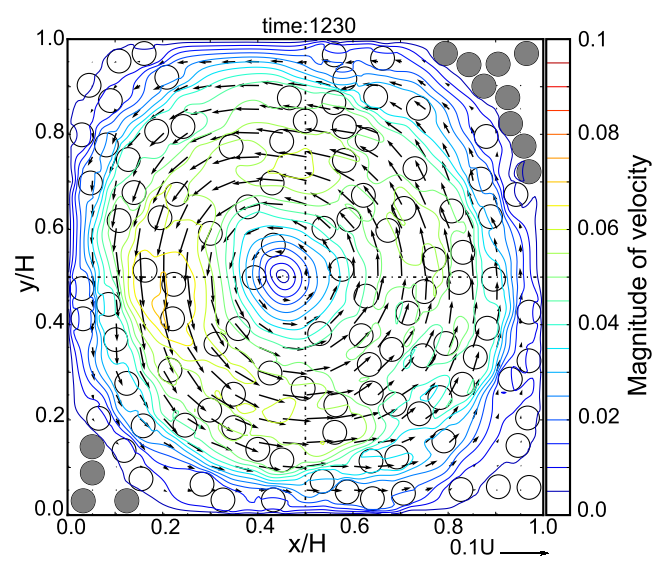

(a) $t U / H=1230$

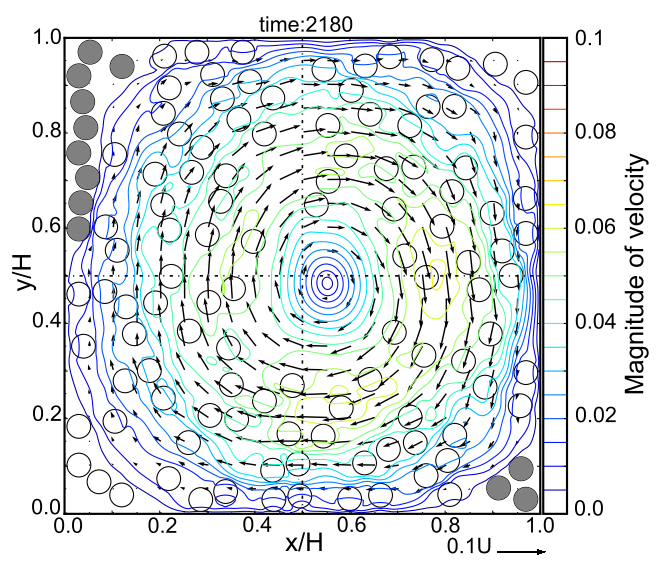

(c) $t U / H=2180$

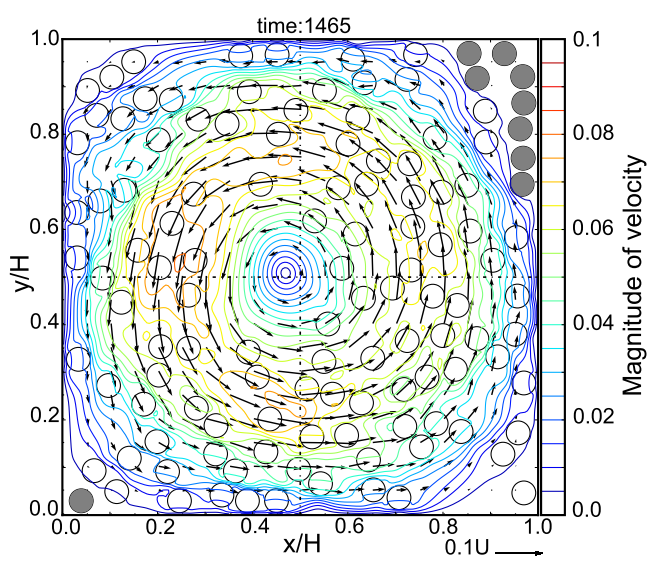

(b) $t U / H=1465$

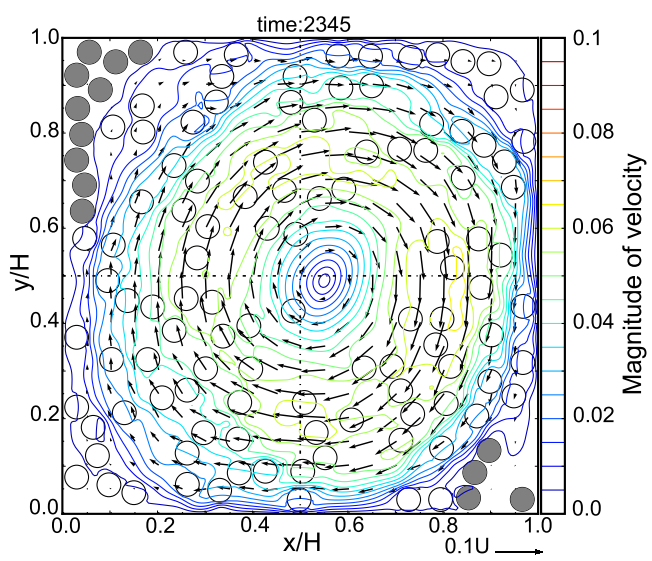

(d) $t U / H=2345$

FIG. 13. Contours of magnitude the velocity at four different instants indicated in Fig. 9. Particles in the region out of the lowest contour of the velocity magnitude are highlighted. Biased presence of particles in the counterconvective corners causes a shift of the circulation center.

Followed by the peak of the WVA index at $t U / H=2180$ (see Fig. 9), for example, the corner MoB approaches zero at time $t U / H=2200$ and the convection becomes weaker. Figure 13(c) shows that the particles in corner 2 cause this attenuation of convection, although the effect of those particles turns out to be not sufficient. In Fig. 15(a), the particles in corners 2 and 3 at time $t U / H=2200$ are colored by aligned groups. The group of particles is determined as follows: if the particles in each corner at $t U / H=2200$ still remain in the same corner after a 20 unit time (at $t U / H=2220$ ), those particles are flagged as the same group, and the particles moved out of the initial (viz. $t U / H=2200$ ) corner region are colored as a different group. The figure shows that, although the particles in corner 2 still persist to weaken the convection, the particles in the driving corners eventually work to boost the convection. The flow field in Figs. 15(c) and 15(d) supports this observation: by the time $t U / H=2220$, some corner particles have been flown away [Fig. 15(c)] and the on-center clockwise vortical flow has been reactivated [Fig. 15(c)], resulting in a stable distribution of MoB [Fig. 15(d)].

The above results suggest that a strong WVA index is not a prerequisite of the reversal. The reversal may not take place if the diagonally opposing corners exhibit too large disparity in the 


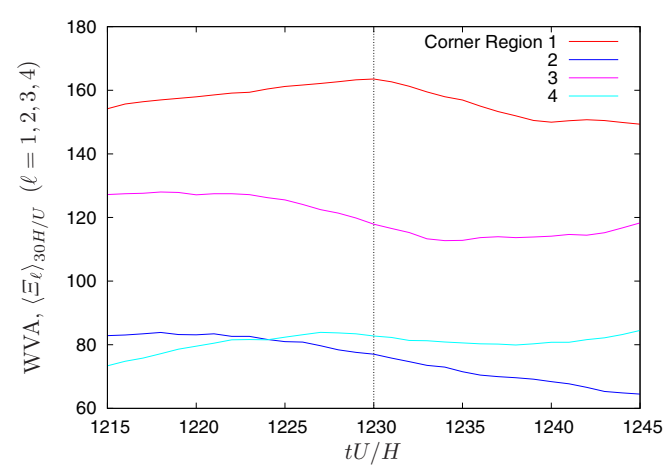

(a)

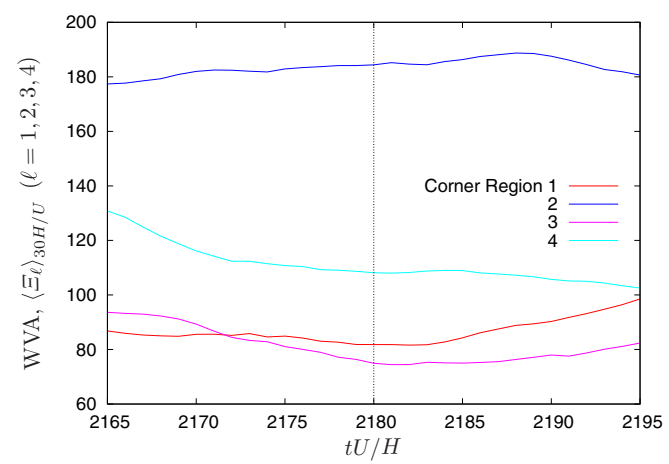

(c)

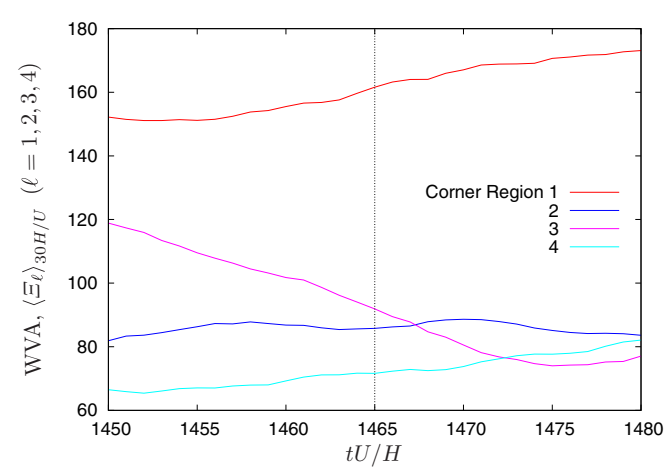

(b)

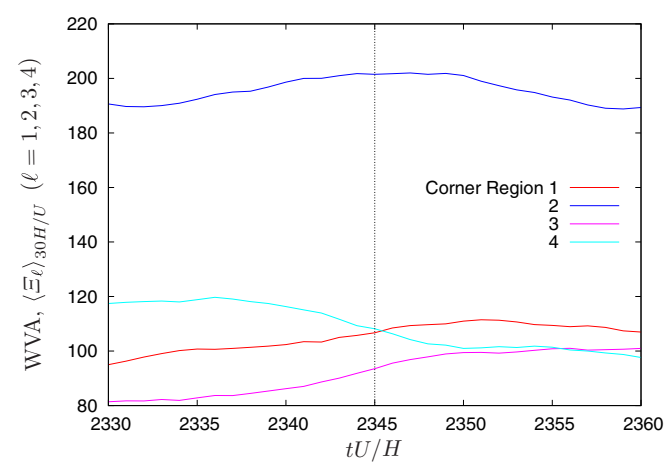

(d)

FIG. 14. The time evolutions of the regional WVA indices around the four instants corresponding to Figs. 13(a)-13(d).

alignment index, and the circulating flow accepts to develop in a rectangular region of an apparently nonunity aspect ratio (due to the off-center shift of the vortex).

\section{EFFECT OF OTHER PARAMETERS}

The mechanism of the particle-driven reversal has become clear by the previous section. However, we have a number of parameters, and a question remains on the parameter range for reversals. In this section, the sensitivity to the major three parameters, $\lambda_{s} / \lambda_{f}, N_{p}$, and $\mathrm{Ra}$, is surveyed to investigate the effects of time scale for heat conduction within a particle, interparticle distance, and buoyancy. Through this survey, we discuss the occurrence condition of the characteristic particle motions.

\section{A. Cases of different $\lambda_{s} / \lambda_{f}$ values}

The conductivity ratio is varied with keeping the number of particles and Rayleigh number in Table I.

Figure 16 shows the time histories of the angular momentum for the following two cases of different conductivity ratios: $\lambda_{s} / \lambda_{f}=10^{1}$ and $10^{3}$. The system with particles of the lower conductivity exhibits a simple circulating flow, although no reversal is observed within the studied time range. The result suggests that the particles of $\lambda_{s} / \lambda_{f}=10^{1}$ cause too weak a temperature change in the corner regions to generate a sufficient counterconvective MoB. On the other hand, the highly conductive particles show the flips of the sign of $L_{z}$, and the flow reversals seem more regular 


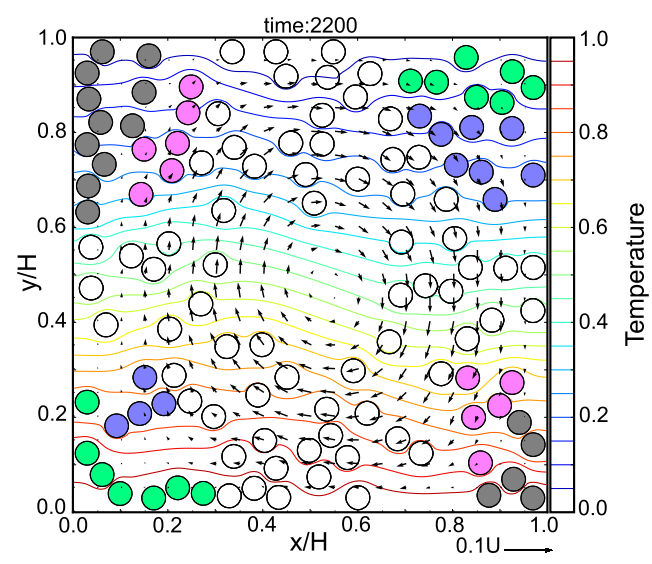

(a)

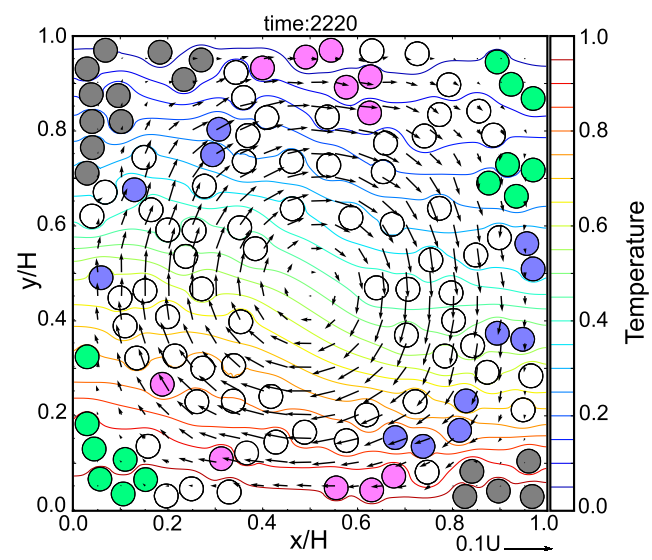

(c)

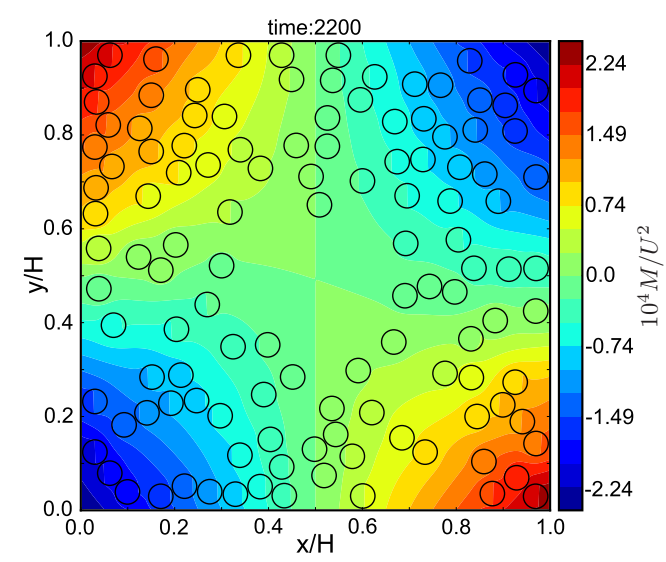

(b)

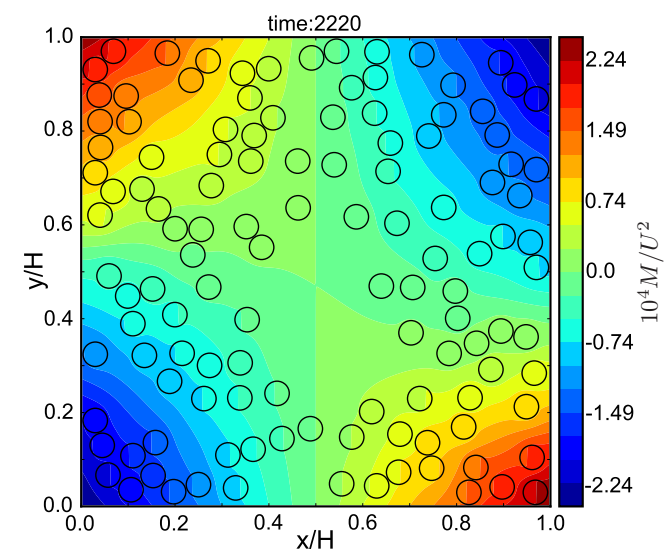

(d)

FIG. 15. The instantaneous velocity and temperature fields at (a) $t U / H=2200$ and (c) $t U / H=2220$, and the corresponding moment of buoyancy at the same times in (b) and (d). The corner particles are colored by groups which are determined as follows: if the particles in each corner at $t U / H=2200$ still remain in the same corner at $t U / H=2220$, those particles are flagged as the same group, and the particles moved out of the corner region are colored as a different group.

than in the previous section (Fig. 2). Our preliminary observation through sequential snapshots gives an impression that the symmetry of the particles are approximately maintained in the case of $\lambda_{s} / \lambda_{f}=10^{3}$ with the amplitude of variation angle much smaller than $2 \pi$. The effect of $\lambda_{s} / \lambda_{f}$ on the oscillation period has been already clarified [14], which will be briefly reviewed later in relation to the analysis of the interparticle spacing.

\section{B. Cases of different initial interparticle distances}

Tsutsumi et al. [16] discussed the effect of the number of particles on the magnitude of convection. They reported that the interparticle spacing influences the development of convection, and, interestingly, increasing the number of particles while keeping the bulk solid volume fraction $20 \%$ causes attenuation of convection (despite the increase in interparticle distances).

Here, with keeping the size of the particles, the number of particles $N_{p}$ is decreased to effectively control the (initial and average) interparticle distances. 


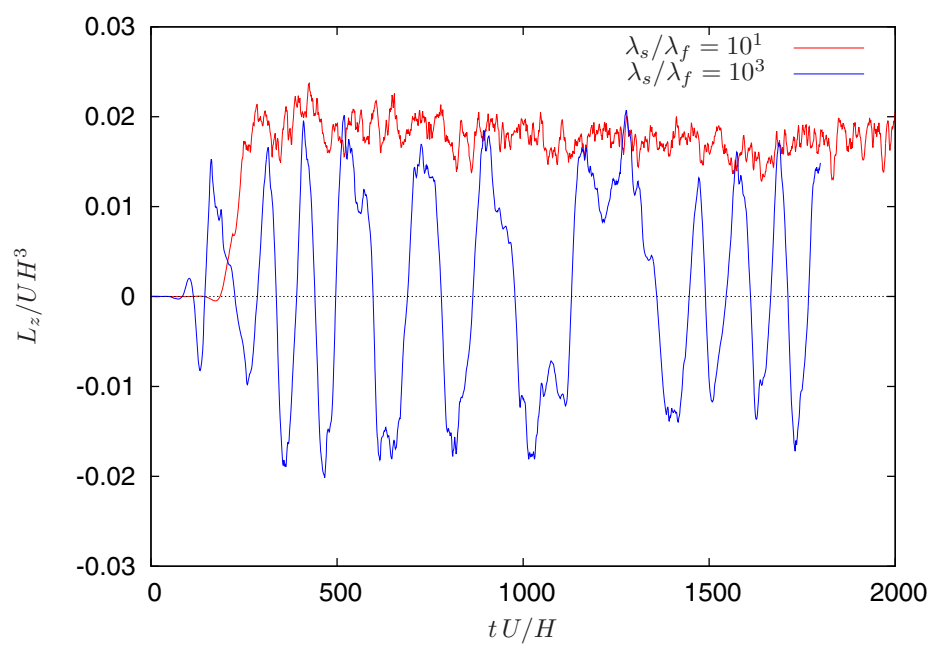

FIG. 16. Time histories of the angular momentum $L_{z}$ for two different $\lambda_{s} / \lambda_{f}$ values: $\lambda_{s} / \lambda_{f}=10^{1}$ and $10^{3}$. The other parameters are kept the same including $N_{p}=11^{2}$ and $\mathrm{Ra}=10^{4}$.

Figure 17 shows the time series of the angular momentum for two different cases of $N_{p}$ in the domain. By increasing the initial interparticle distances with $N_{p}=10^{2}$, reversal disappears and a circulating flow evolves, whereas a regular symmetric oscillation mode appears by decreasing the interparticle spacing $\left(N_{p}=12^{2}\right)$.

A limiting value of $N_{p}$ for the transition from circulation is estimated in the following. With $\sqrt{N_{p}}$ particles along one side of the square domain $H$, the interparticle (surface-to-surface) distance is

$$
\Delta l_{\mathrm{ss}}=\frac{H}{\sqrt{N_{p}}}-D_{p} .
$$

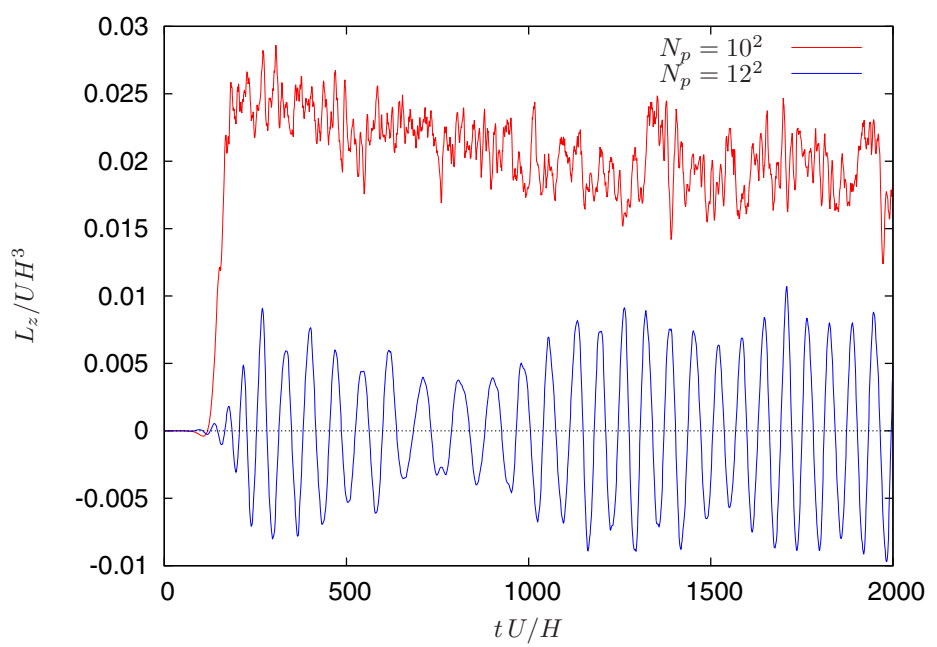

FIG. 17. Time histories of the angular momentum $L_{z}$ for two different $N_{p}$ values: $N_{p}=10^{2}$ and $12^{2}$. The other parameters are kept the same including $\lambda_{s} / \lambda_{f}=10^{2}$ and $\mathrm{Ra}=10^{4}$. 


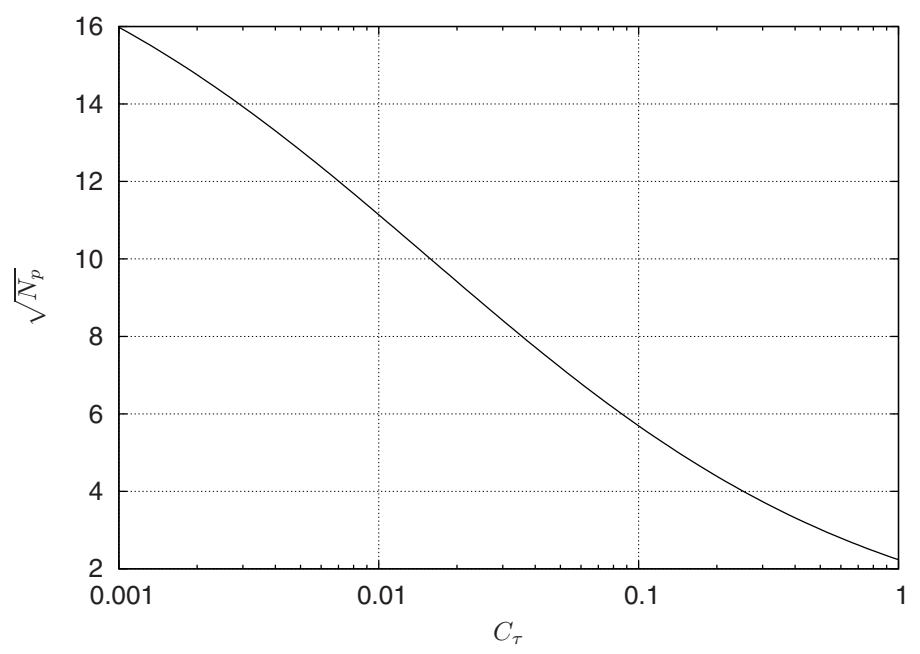

FIG. 18. Estimated lower bound of $\sqrt{N_{p}}$ as a function of $C_{\tau}$ for the parameter values given in Table I and $C_{R}=10^{1}$.

The time scale $\tau_{\mathrm{d}}$ at which the heat is conducted to the distance $\Delta l_{\mathrm{ss}}$ is

$$
\tau_{\mathrm{d}}=\frac{\left(\Delta l_{\mathrm{ss}}\right)^{2}}{\lambda_{f} /(\rho c)_{f}} \text {. }
$$

Assuming that the oscillation is maintained unless the thermal boundary layer around each particle is blown away by the convection due to the particle oscillation, we postulate a necessary condition for maintaining the oscillation such that the linear temperature profile (in the fluid phase) is restored within a fraction of oscillation period. Here, a half period is assumed as the longest possible fraction (due to the antiphase motion for the two particles of the point symmetry with respect to the domain center), then the condition is given as

$$
\tau_{\mathrm{d}} \ll \frac{\tau_{R}}{2},
$$

where $\tau_{R}$ is the oscillation period modeled as follows [14]:

$$
\frac{\tau_{R}}{\tau_{\mathrm{ref}}}=C_{R}\left(\frac{\lambda_{s}}{\lambda_{f}} \frac{(\rho c)_{s}}{(\rho c)_{f}}\right)^{1 / 4} .
$$

Here, the reference time $\tau_{\text {ref }}$ is given as

$$
\tau_{\text {ref }}=\frac{H}{U}=\frac{H^{2}}{v_{f}}\left(\frac{\mathrm{Pr}}{\mathrm{Ra}}\right)^{1 / 2}
$$

and $C_{R}$ is a constant. The result in Ref. [14] suggests $C_{R} \approx 10^{1}$. Here, to calculate the lower bound of $N_{p}$, Eq. (19) is modified to

$$
\tau_{\mathrm{d}} \approx C_{\tau} \frac{\tau_{R}}{2}
$$

where $C_{\tau}$ is a scaling constant. Then, the necessary condition for the interparticle distance to maintain the oscillation reads

$$
\sqrt{N_{p}} \approx\left[\frac{D_{p}}{H}+\left(\frac{C_{\tau} C_{R}}{2}\right)^{1 / 2}\left(\frac{1}{\operatorname{Pr} \mathrm{Ra}}\right)^{1 / 4}\left(\frac{\lambda_{s}}{\lambda_{f}} \frac{(\rho c)_{s}}{(\rho c)_{f}}\right)^{1 / 8}\right]^{-1}
$$




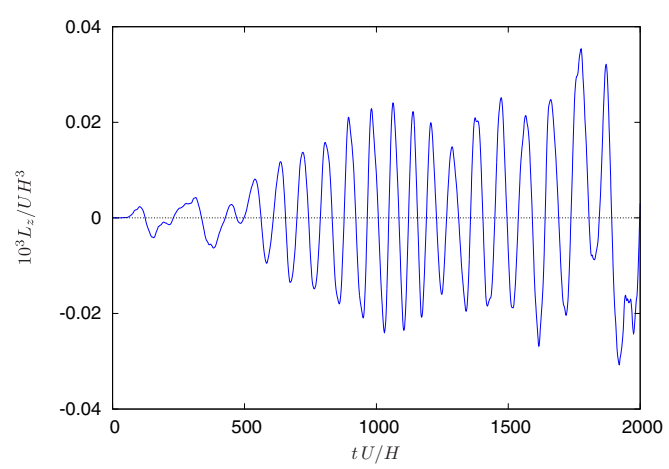

(a)

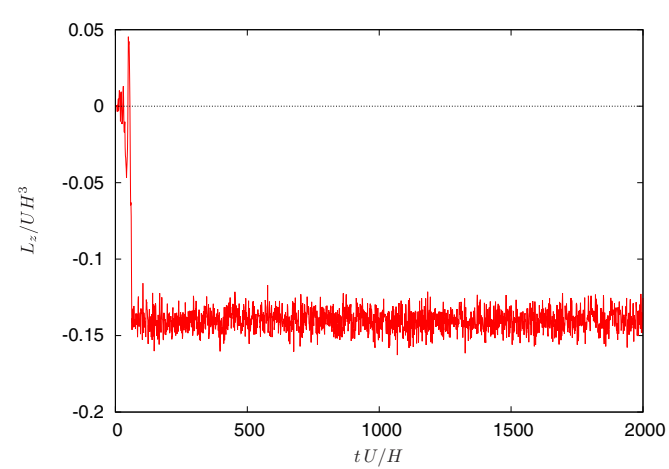

(b)

FIG. 19. Time evolution of the angular momentum $L_{z}$ for two different Ra values: Ra $=5000$ and $10^{5}$. The other parameters are kept the same including $\lambda_{s} / \lambda_{f}=10^{2}$ and $N_{p}=11^{2}$.

This is the lower bound of the number of particles to cause regular symmetric oscillation. Figure 18 plots Eq. (23) against $C_{\tau}$ with the other parameter values given in Table I. Considering $\tau_{R} / \tau_{\text {ref }} \approx 10^{1}$ in the initial linear-developing regime from Ref. [14], the order of magnitude for $C_{\tau}\left(=2 \tau_{d} / \tau_{R}\right)$ is evaluated to be $10^{-2}$. From the figure, the estimated lower bound of $\sqrt{N_{p}}$ is not very sensitive to $C_{\tau}$ around $C_{\tau}=10^{-2}$, and the above equation (23) approximately explains the transition between $10^{2} \leqslant N_{p} \leqslant 12^{2}$ in Fig. 17. Note that, by increasing the number of particles (i.e., decreasing the interparticle spacing) to $N_{p}=13^{2}$ or $14^{2}$, the system exhibits more clear regular symmetric oscillation modes. However, with further increase of the number of particles in a closed domain, particle-dispersed natural convection shows a slow suspension flow mode (with hindered heat transfer) and has less possibility to develop into a circulating or oscillating mode, as Gu et al. [15] reported.

\section{Cases of different Ra}

The effect of Ra is summarized in Fig. 19. At Ra $=5000$, Fig. 19(a) shows a regular oscillation, but the amplitude of the angular momentum is small. On the other hand, the system of $\mathrm{Ra}=10^{5}$ exhibits a large-scale circulating flow.

Equations (20) and (21) suggest that the oscillation period is proportional to $\mathrm{Ra}^{-1 / 2}$, and our preliminary study confirms approximately this trend of $\tau_{R}$.

\section{CONCLUSIONS}

Numerical simulation of particle-dispersed natural convection in a square domain was carried out, and the mechanism of flow reversal was revealed. Focusing on the corner regions of the domain, we confirmed that the moment of buoyancy generated in the corner region (corner MoB) is important for determining the direction of convection. Through the analysis of the effect of the vertical alignments of the particles in the corner regions, the corner MoB was found to be typically generated in the counterconvective direction by the transported particles, which cause an apparent increase in thermal conductivity in the corner regions, resulting in the reversal of convection.

The proposed weighted vertical alignment index is a good indicator to explain the reversals. The study showed that the vertical assemblies of particles in a pair of diagonal corners are essential to trigger reversals: a large disparity in the alignment index between the diagonally opposing corners does not overturn the flow, but a single roll structure survives with an off-center shift.

The illustrated mechanism is different from the reversal mechanisms in the single-phase RB convection: the multiphase reversal events result from a dynamic system driven by local buoyancy 
distribution induced by unevenly distributed particles, and, for this reason, interpretation of the reversals as a homogeneous isotropic suspension may not work.

Varying the major three parameters suggested that the nonregular asymmetric reversal mode takes place in a small region of the parameter space, particularly of the interparticle spacing. The survey has posed remaining problems for identifying the boundary between the regular symmetric oscillation and the nonregular asymmetric reversal modes, which is the subject of ongoing study by the present authors.

\section{ACKNOWLEDGMENTS}

This work was partly supported by Grant-in-Aid (B) No. 17H03174 of the Japan Society for the Promotion of Science (JSPS). One of the authors, S.T., also gratefully acknowledges the financial support of the Kurata Grants No. 871 by the Hitachi Global Foundation.

\section{APPENDIX: WEIGHTED VERTICAL ALIGNMENT (WVA)}

We construct an approximate model of heat flux between a pair of particles $i$ and $j$ in a corner region. The particles and the interparticle region are modeled with a rectangular object of composite materials of different heat conductivities, and the temperature distribution in a fluid is assumed to be linear around the particles. A schematic is shown in Fig. 20.

The apparent conductivity $\lambda$ of the rectangular object along the center-to-center direction is modeled to be

$$
\frac{\left|\boldsymbol{r}_{i, j}\right|}{\lambda} \simeq \frac{D_{p} / 2}{\lambda_{s}}+\frac{\left|\boldsymbol{r}_{i, j}\right|-D_{p}}{\lambda_{f}}+\frac{D_{p} / 2}{\lambda_{s}},
$$

where $\boldsymbol{r}_{i, j}=\boldsymbol{x}_{j}^{p}-\boldsymbol{x}_{i}^{p}$ is the relative vector from the center of particle $i$ to that of particle $j$. With this conductivity, the heat flux between the two particles is given as follows:

$$
\left|\boldsymbol{q}_{i, j}\right| \simeq \lambda \frac{\delta T}{\left|\boldsymbol{r}_{i, j}\right|} \simeq\left(\frac{D_{p}}{\lambda_{s}}+\frac{\left|\boldsymbol{r}_{i, j}\right|-D_{p}}{\lambda_{f}}\right)^{-1}\left(\frac{\Delta T}{H} \boldsymbol{r}_{i, j} \cdot \boldsymbol{e}_{y}\right),
$$

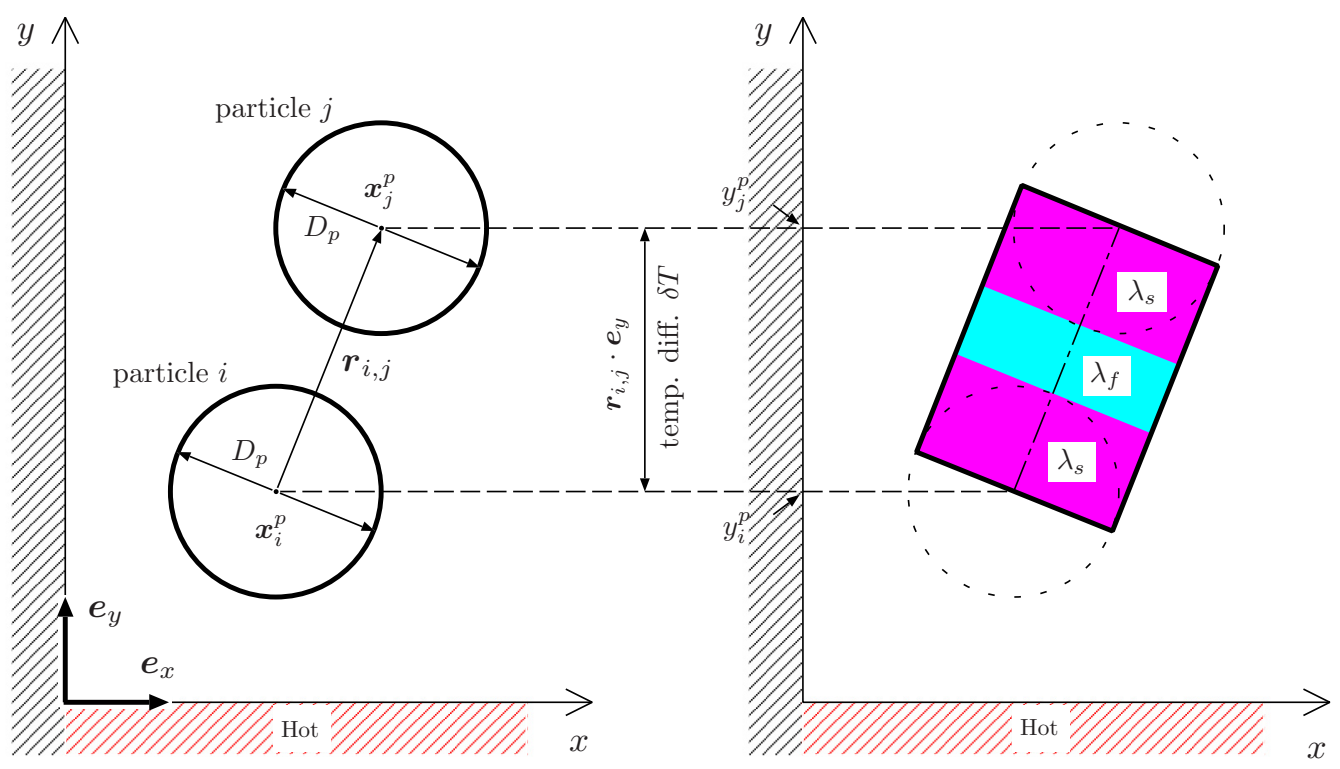

FIG. 20. Schematic of a two-particle system and a replaced composite material. 
where $\boldsymbol{e}_{y}$ is the unit vector in the $y$ direction, and the normalized heat flux between the particles is as follows:

$$
\left(\lambda_{f} \frac{\Delta T}{H}\right)^{-1}\left|\boldsymbol{q}_{i, j}\right| \simeq\left(\frac{\lambda_{f}}{\lambda_{s}}+\frac{\left|\boldsymbol{r}_{i, j}\right|-D_{p}}{D_{p}}\right)^{-1}\left(\frac{\boldsymbol{r}_{i, j}}{D_{p}} \cdot \boldsymbol{e}_{y}\right) .
$$

Here, we introduce a vertical alignment of the two particles in terms of the conductive heat flux as

$$
\xi_{i, j}=w_{i, j}^{\mathrm{d}}\left|\frac{\boldsymbol{r}_{i, j}}{D_{p}} \cdot \boldsymbol{e}_{y}\right|,
$$

where the weight $w_{i, j}^{\mathrm{d}}$ is defined as follows:

$$
w_{i, j}^{\mathrm{d}}{ }^{-1}=\left(\frac{\lambda_{s}}{\lambda_{f}}\right)^{-1}+\max \left[\frac{\left|\boldsymbol{r}_{i, j}\right|}{D_{p}}-1,0\right] .
$$

Here, the max function is introduced to avoid an unreasonable decrease of the weight value in the case of contacting particles. Then, the weighted vertical alignment (WVA) of the particles in corner region $\ell$ is defined as the sum of the interparticle alignments in the region:

$$
\Xi_{\ell}=\sum_{\substack{\left|\boldsymbol{r}_{i, j}\right|<2 D_{p} \\ \boldsymbol{r}_{i, j} \cdot \boldsymbol{e}_{\boldsymbol{y}}>0 \\ \text { in region } \ell}} \xi_{i, j}
$$

and the collective effect of the WVAs in the corner regions on the flow reversal is evaluated by the following cross-coupled WVA index:

$$
\Xi=\left(\Xi_{2}+\Xi_{4}\right)-\left(\Xi_{1}+\Xi_{3}\right)
$$

The above model does not include the effect of convection, and therefore, the nondimensionalized heat flux [Eq. (A1)] may underestimate the heat flux. Introducing the characteristic velocity at the spatial scale of particle diameter as

$$
\begin{aligned}
U^{\prime} & =\sqrt{g \beta \delta T D_{p}}=\sqrt{g \beta \Delta T H} \sqrt{\frac{\delta T}{\Delta T} \frac{D_{p}}{H}} \\
& =U \sqrt{\frac{\boldsymbol{r}_{i, j} \cdot \boldsymbol{e}_{y}}{H} \frac{D_{p}}{H}},
\end{aligned}
$$

the effect of the convection is incorporated into the above model as

$$
\left|\boldsymbol{q}_{i, j}\right| \simeq \lambda \frac{\delta T}{\left|\boldsymbol{r}_{i, j}\right|} \pm \rho c U^{\prime} \delta T=\left(\frac{\lambda}{\left|\boldsymbol{r}_{i, j}\right|} \pm \rho c U^{\prime}\right)\left(\frac{\Delta T}{H} \boldsymbol{r}_{i, j} \cdot \boldsymbol{e}_{y}\right) .
$$

The \pm sign of the convective term is determined by whether the flow is clockwise or counterclockwise direction. Finally, the regional WVA index is defined as follows:

$$
\Xi_{\ell}=\sum\left(\lambda_{f} \frac{\Delta T}{H}\right)^{-1}\left|\boldsymbol{q}_{i, j}\right|=\sum w_{i, j}^{\mathrm{d}+\mathrm{v}}\left|\frac{\boldsymbol{r}_{i, j}}{D_{p}} \cdot \boldsymbol{e}_{y}\right|,
$$

where the summation is taken for the same condition as in Eq. (A3) and

$$
\begin{aligned}
w_{i, j}^{\mathrm{d}+\mathrm{v}} & =w_{i, j}^{\mathrm{d}}+s \sqrt{\operatorname{Ra} \operatorname{Pr} \frac{\boldsymbol{r}_{i, j} \cdot \boldsymbol{e}_{y}}{H}\left(\frac{D_{p}}{H}\right)^{3} \Upsilon_{i, j},} \\
s & =\operatorname{sgn}\left[L_{z}\right] \operatorname{sgn}\left[x-\frac{H}{2}\right], \\
\Upsilon_{i, j} & =\frac{\left|\boldsymbol{u}\left(t, x_{c}, y_{c}\right)\right|}{\max _{x, y, t}|\boldsymbol{u}|}
\end{aligned}
$$




$$
\begin{aligned}
& x_{c}=\frac{\boldsymbol{x}_{i}^{p}+\boldsymbol{x}_{j}^{p}}{2} \cdot \boldsymbol{e}_{x}, \\
& y_{c}=\frac{\boldsymbol{x}_{i}^{p}+\boldsymbol{x}_{j}^{p}}{2} \cdot \boldsymbol{e}_{y},
\end{aligned}
$$

where $\boldsymbol{e}_{x}$ is the unit vector in the $x$ direction. With the above combined weight $w_{i, j}^{\mathrm{d}+\mathrm{v}}$ covering both conduction and convection, the cross-coupled effect is given as Eq. (A4).

[1] S. Chandrasekhar, Hydrodynamic and Hydromagnetic Stability (Dover, New York, 1981).

[2] E. L. Koschmieder, Bénard Cells and Taylor Vortices (Cambridge University Press, Cambridge, UK, 1993).

[3] K. R. Sreenivasan, A. Bershadskii, and J. J. Niemela, Mean wind and its reversal in thermal convection, Phys. Rev. E 65, 056306 (2002).

[4] K. Sugiyama, R. Ni, R. J. A. M. Stevens, T. S. Chan, S.-Q. Zhou, H.-D. Xi, C. Sun, S. Grossmann, K.-Q. Xia, and D. Lohse, Flow Reversals in Thermally Driven Turbulence, Phys. Rev. Lett. 105, 034503 (2010).

[5] M. Chandra and M. K. Verma, Dynamics and symmetries of flow reversals in turbulent convection, Phys. Rev. E 83, 067303 (2011).

[6] M. Chandra and M. K. Verma, Flow Reversals in Turbulent Convection via Vortex Reconnections, Phys. Rev. Lett. 110, 114503 (2013).

[7] A. Castillo-Castellanos, A. Sergent, and M. Rossi, Reversal cycle in square Rayleigh-Bénard cells in turbulent regime, J. Fluid Mech. 808, 614 (2016).

[8] B. Podvin and A. Sergent, A large-scale investigation of wind reversal in a square Rayleigh Bénard cell, J. Fluid Mech. 766, 172 (2015).

[9] R. Benzi, Flow Reversal in a Simple Dynamical Model of Turbulence, Phys. Rev. Lett. 95, 024502 (2005).

[10] R. Benzi and R. Verzicco, Numerical simulations of flow reversal in Rayleigh-Bénard convection, Europhys. Lett. 81, 64008 (2008).

[11] B. Gallet, J. Herault, C. Laroche, F. Pétrélis, and S. Fauve, Reversals of a large-scale field generated over a turbulent background, Geophys. Astrophys. Fluid Dyn. 106, 468 (2012).

[12] F. F. Araujo, S. Grossmann, and D. Lohse, Wind Reversals in Turbulent Rayleigh-Bénard Convection, Phys. Rev. Lett. 95, 084502 (2005).

[13] C. Resagk, R. du Puits, A. Thess, F. V. Dolzhansky, S. Grossmann, F. Fontenele Araujo, and D. Lohse, Oscillations of the large scale wind in turbulent thermal convection, Phys. Fluids 18, 095105 (2006).

[14] S. Takeuchi, T. Tsutsumi, and T. Kajishima, Effect of temperature gradient within a solid particle on the rotation and oscillation modes in solid-dispersed two-phase flows, Int. J. Heat Fluid Flow 43, 15 (2013).

[15] J. Gu, S. Takeuchi, and T. Kajishima, Influence of rayleigh number and solid volume fraction in particledispersed natural convection, Int. J. Heat Mass Transfer 120, 250 (2018).

[16] T. Tsutsumi, S. Takeuchi, and T. Kajishima, Heat transfer and particle behaviours in dispersed two-phase flow with different heat conductivities for liquid and solid, Flow, Turbul. and Combust. 92, 103 (2014).

[17] T. Kajishima and S. Takiguchi, Interaction between particle clusters and particle-induced turbulence, Int. J. Heat Fluid Flow 23, 639 (2002).

[18] S. Takeuchi, T. Tsutsumi, K. Kondo, T. Harada, and T. Kajishima, Heat transfer in natural convection with finite-sized particles considering thermal conductance due to inter-particle contacts, Comput. Thermal Sci. 7, 385 (2015).

[19] Y. Tsuji, T. Kawaguchi, and T. Tanaka, Discrete particle simulation of two-dimensional fluidized bed, Powder Technol. 77, 79 (1993). 